\title{
Turbulence structure in a diabatically heated forest canopy composed of fractal Pythagoras trees
}

Received: 24 December 2011 / Accepted: 23 August 2012 / Published online: 26 September 2012

(C) Springer-Verlag 2012

\begin{abstract}
We investigate the turbulent flow through a heterogeneous forest canopy by high-resolution numerical modeling. For this purpose, a novel approach to model individual trees is implemented in our large-eddy simulation (LES). A group of sixteen fractal Pythagoras trees is placed in the computational domain and the tree elements are numerically treated as immersed boundaries. Our objective is to resolve the multiscale flow response starting at the diameter of individual tree elements up to the depth of the atmospheric surface layer. A reference run, conducted for the forest flow under neutral thermal stratification, produces physically meaningful turbulence statistics. Our numerical results agree quantitatively with data obtained from former field-scale LESs and wind tunnel experiments. Furthermore, the numerical simulations resolve vortex shedding behind individual branches and trunks as well as the integral response of the turbulent flow through the heterogeneous forest canopy. A focus is the investigation of the turbulence structure of the flow under stable thermal stratification and in response to the heating of the fractal tree crowns. For the stratified flows, statistical quantities, e.g. turbulent kinetic energy and vorticity, are presented and the turbulent exchange processes of momentum and heat are considered in detail. The onset and formation of coherent structures such as elevated shear layers above the diabatically heated forest canopy are analyzed. For the stably stratified flow, temperature ramps above the forest canopy were simulated in agreement with previous observations. Thermally driven vortices with a typical diameter of the canopy height were simulated when the tree crowns were diabatically heated. The impact of the coherent flow structures on the heat flux is investigated.
\end{abstract}

Keywords Atmospheric boundary layer - Forest canopy · Fractal trees - Heat - Turbulence

\section{Introduction}

Flow through forest canopies transports momentum, heat, aerosol particles and trace gases as carbon dioxide, oxygen and water vapor. The associated atmospheric exchange processes between the forest and the atmosphere occur on a broad range of scales. Photosynthesis and transpiration on the leaves [32] are examples representing

Communicated by R. Klein.

J. Schröttle $(\varangle)$

Meteorologisches Institut der Universität München (LMU),

Theresienstr. 47, 80333 Munich, Germany

E-mail: josef.schroettle@ physik.uni-muenchen.de

A. Dörnbrack

Institut für Physik der Atmosphäre, DLR Oberpfaffenhofen,

82234 Weßling, Germany

E-mail: andreas.doernbrack@dlr.de

Tel.: ++49/8153/28-2588

Fax: $++49 / 8153 / 28-1841$ 
the small-scale end of the spectrum. Wake vortices can be found behind individual branches and trunks $[3,8,13]$, whereas the largest turbulent eddies scale with the depth of the atmospheric surface layer.

Atmospheric transport is fundamentally nonlinear. In flows through forest canopies, it generates coherent vortices, leads to scale-contractions as in shear layers above the trees, and is subject to buoyancy effects [45,61]. The essentially multiscale exchange processes can effectively be influenced by diabatic effects $[21,46,61]$ at the scale of the tree crowns [58]. As stated in a recent review by Belcher et al. [2]: the impact of these diabatic processes on the 3D turbulence structure, especially under stable thermal stratification, has been insufficiently quantified for forest canopies. Further knowledge about the turbulence above and within forest canopies is necessary for practical applications such as regional climate prediction, weather forecasting, as well as for the analysis and interpretation of micro-meteorological measurements [18].

Given the nonlinearity of the exchange processes and their multiscale nature there are two principal options for their quantification: field or laboratory measurements and reliable numerical simulations. There is a long series of field campaigns investigating turbulent exchange processes for various forest stands, e.g. for a tropical rainforest [7]. The observed turbulence statistics, as for momentum flux, depends quantitatively on the canopy geometry and the corresponding leaf area index: LAI ("Appendix A"). Field campaigns were conducted to study the forest flow under different atmospheric conditions [7,21,33,46,55,57]. For example, so-called thermal ramps were discovered above the forest canopy under stable thermal stratification [21]. These tilted, coherent ejections of cold air are generated by the mechanical force of the wind inside the forest canopy.

During the recent field campaign CHATS 2007, ${ }^{1}$ turbulent transport processes and coherent structures were investigated by high-resolution remote-sensing instruments, e.g. by a Doppler lidar [33]. The local temperature field proved to be heterogeneous within the forest canopy leading to large gradients of $\approx 5 \mathrm{~K}$ within less than one meter. Heterogeneities in the temperature field can occur at even smaller scales of individual branches. ${ }^{2}$ The CHATS 2007 observations confirm earlier measurements of warmer tree crowns compared to the trunk space during the day.

The later result agrees with observations of the EAGLE 2006 campaign, ${ }^{3}$ where in the mean the tree crown was about $3 \mathrm{~K}$ warmer than the ambient air. Inter alia, this campaign investigated the vertical moisture and temperature distributions by means of instrumented towers up to the height of the surface layer [57]. The long-term, ground-based monitoring stations of the FLUXNET project ${ }^{4}$ observe and document the exchange of carbon dioxide, water vapor, and energy between terrestrial ecosystems and the atmosphere at regional and global scales [1].

As already stated by Shaw after analyzing field experiments in the late 1980s, the proper interpretation of such observations requires an accurate representation of "the aerodynamic characteristics of the forest" [46]. Besides dynamical processes, flow variations due to diabatic effects and the impact of the background stratification must be considered as well. For this purpose, numerical simulations of different complexity were conducted. Early numerical approaches, as the pioneering large-eddy simulation (LES) in 1992, conducted by Shaw and Schumann [47], investigated the turbulence structure above a forest canopy. The impact of the forest on the flow was represented by an external drag force which is proportional to a vertically varying, yet horizontally uniform leaf area density: $\operatorname{LAD}(z)$ ("Appendix A"). This approach which does not resolve the individual forest elements but models their integral effect is sometimes referred to as "field-scale approach" [59].

There are numerous numerical simulations employing the same ansatz as proposed by [47] for diverse research applications and forest topologies [15]. Over the years, numerical simulations gradually approached finer scales and forest heterogeneities were treated by spatially varying drag forces. Recently, heterogeneities of the forest canopy were studied by LESs, e.g. the flow over forest edges [12]. The impact of smaller scale heterogeneities in a patchy forest with horizontally variable leaf area density and a certain stem volume on the scale of $1 \mathrm{~m}$ was investigated in [3]. Although these simulations already approach the bulk of the tree scale, they do not resolve the geometry of individual tree elements. An early attempt to investigate canopy flow at this scale were Reynolds-averaged Navier-Stokes (RANS) simulations, e.g. as conducted by [23]. Single trees were modeled with smooth cylindrical trunks, as well as bowl- and cone-shaped tree crowns. However, trees

\footnotetext{
1 The Canopy Horizontal Array Turbulence Study field experiment (CHATS) was conducted in an orchard canopy near Dixon, CA, USA from 15 March to 15 June 2007; see http://www.eol.ucar.edu/isf/projects/CHATS/isff.

${ }^{2}$ See infrared pictures at http://www.eol.ucar.edu/deployment/field-deployments/field-projects/chats-project/photos/thermalcamera-images.

${ }^{3}$ EAGLE 2006: Multi-purpose, multi-angle and multi-sensor in-situ and airborne campaigns over grassland and forest.

${ }^{4}$ http://daac.ornl.gov/FLUXNET/fluxnet.shtml.
} 
are archetypical fractals. Modeling trees with fractal geometric shapes alters the topological properties of a virtual forest ${ }^{5}$ and of the resulting turbulent flow.

Recently, [9] simulated the flow structure induced by a single tree using LES. The numerical simulation of the flow around an individual tree resolving the trunk and branches is sometimes referred to as "plantscale approach" [59]. In [9] the tree was represented in different fractal shapes with up to 3 resolved branch generations. Their simulations produce small-scale turbulence in the lee of the tree. The associated drag coefficient of the flow converges with the number of branch generations. To our knowledge, no other simulation of the flow through fractal trees has been undertaken so far. A multiscale simulation including relatively small wake vortices in the flow structure and by the same time providing the integral effect of the forest canopy on the flow has not been conducted until now.

There are only few studies investigating the impact of heat on the flow structure. Interestingly, in their seminal paper, Shaw and Schumann [47] already assumed an exponential decline of the horizontally homogeneous heat flux inside the canopy, representing the effect of the vertical distribution of net radiation. This idea has yet not been used for systematic studies investigating the impact of diabatic processes on the turbulence structure of forest flows. Just recently, this approach was applied [3] to simulate two different scenarios: one represents the winter season with strong winds above a sparse canopy and a slightly unstable boundary layer; the other represents the spring season with a dense canopy, calm conditions, and a strongly convective boundary layer.

To our knowledge, former LESs are restricted to neutral or slightly unstable thermal conditions. Complementary wind tunnel experiments to validate LESs of canopy flows are only available for neutral conditions $[6,16,41]$. Additionally, only a few wind tunnel experiments consider forest canopy turbulence, most concentrate on smaller scale canopies, e.g. crop fields, waving wheat, or the bending of individual tree crowns $[6,15,10]$. No LES results were published about a forest canopy flow for a stable stratification to confirm experimental results of e.g. [21].

Here, our objective is to investigate the flow response induced by a cluster of discrete, diabatically heated fractal trees. Simulating the combined effect of a multitude of trees is an extension of the LESs as conducted in [9]. For this purpose, a grove consisting of sixteen fractal Pythagoras trees ${ }^{6}$ was composed. The tree elements are treated as immersed boundaries in the numerical model $[22,30,53,54]$. Our numerical simulations cover all significant spatial scales starting with the depth of the surface layer of $\approx 100 \mathrm{~m}$, approaching the tree height of about $10 \mathrm{~m}$, and eventually resolving individual branch geometry with a resolution of $\approx 5 \mathrm{~cm}$. Furthermore, the air in the crown space is diabatically heated by branches which are prescribed to be warmer than the ambient air consistent with recent observations. So far, it is an open question whether the turbulent flow through such a diabatically heated, fractal grove can be successfully simulated across this large range of scales with a current state-of-the-art LES model. Following this approach, we aspire to bridge the gap between local observations inside the forests and former field-scale simulations by means of high-resolution numerical simulations.

The paper is organized as follows. Section 2 gives a summary of the tools that are employed for our numerical simulations: the flow solver EULAG [39], the setup of the numerical experiments, and the methods for analyzing the data. Section 3 provides the results of the reference simulation for the well-documented neutral canopy flow and compares our results with former LES and wind tunnel studies. Diabatic effects on the simulated flow structure are investigated in Sect. 4 and the turbulence structure above the diabatically heated tree crowns is portrayed in Sect. 5. Discussions and conclusions close this paper in Sect. 6.

\section{Method}

\subsection{Numerical model}

Our numerical simulations were conducted with the geophysical fluid solver EULAG, that has proven its abilities for simulating thermo-fluid flows across a wide range of scales and diverse physical scenarios ranging from solar convection to laboratory experiments [39]. Here, we consider small-scale boundary-layer flows and thus, the incompressible Boussinesq approximation (1) is adopted. For the forest canopy flow the governing equations are compactly written in Cartesian coordinates as follows:

\footnotetext{
5 "Clouds are not spheres, mountains are not cones, coastlines are not circles, and bark is not smooth, nor does lightning travel in a straight line." [28].

${ }^{6}$ http://demonstrations.wolfram.com/PythagorasTree/.
} 


$$
\begin{aligned}
\nabla \cdot \mathbf{v} & =0, \\
\frac{\mathrm{d} \mathbf{v}}{\mathrm{d} t} & =-\nabla \pi^{\prime}-\mathbf{g} \frac{\theta^{\prime}}{\theta_{b}}+\mathcal{D}^{\mathbf{v}}-\beta\left(\mathbf{v}-\mathbf{v}_{F}\right), \\
\frac{\mathrm{d} \theta^{\prime}}{\mathrm{d} t} & =-\mathbf{v} \cdot \nabla \theta_{e}+\mathcal{D}^{\theta}-\beta\left(\theta-\theta_{F}\right), \\
\frac{\mathrm{d} e}{\mathrm{~d} t} & =\mathcal{S}(e)-\beta\left(e-e_{F}\right) .
\end{aligned}
$$

Here, $\mathrm{d} / \mathrm{d} t$ denotes the material derivative, $\mathbf{v}$ the velocity vector, $\pi^{\prime}=\left(p-p_{e}\right) / \rho_{b}$ with the pressure $p$, and the constant Boussinesq reference density $\rho_{b}=1.128 \mathrm{~kg} \mathrm{~m}^{-3}$. The gravitational acceleration is $\mathbf{g}=$ $(0,0,-g)$. The quantity $\theta^{\prime}$ represents the deviation of the potential temperature $\theta$ from a hydrostatically balanced environmental atmospheric state $\theta_{e}$, where $\theta_{e}(z)=\theta_{b}\left(1+N^{2} z / g\right)$ with the Brunt-Väisälä frequency $N$ and the Boussinesq reference state $\theta_{b}=300 \mathrm{~K}$.

The subgrid-scale terms in Eqs. (2) and (3) are $\mathcal{D}^{\mathbf{v}}:=\nabla \boldsymbol{\tau}$, where $\boldsymbol{\tau}$ is the deviatoric stress tensor and $\mathcal{D}^{\theta}:=\nabla \cdot \mathcal{H}$ with heat flux $\mathcal{H} \sim \nabla \theta$. We employ a Smagorinski-type turbulence closure based on the prognostic subgrid-scale turbulent kinetic energy (TKE) Eq. (4); see [29] and Sect. 3.1 of [35] for a succinct but comprehensive description of the TKE model. The subgrid-scale parameterizations used for our simulations were validated for a broad range of flow regimes, such as gravity waves over mountains or a convective boundary layer $[11,29,52]$.

The relaxation terms including $\beta(\mathbf{x})$ on the rhs of Eqs. (2)-(4) represent additional forcings due to the presence of the virtual forest canopy. The basis of our numerical approach to model the fractal trees is the immersed boundary (IMB) method [22,30], where solid objects are explicitly resolved by the high-resolution numerical simulations. The flow experiences the IMBs by velocity stagnation or by a temperature contrast within the solid medium and, consequently, shear and temperature gradients develop in the flow. In Eqs. (2)-(4), the states inside the forest elements are denoted by the subscript $F_{F}$; a natural choice for solid, non-moving obstacles is $\mathbf{v}_{F} \equiv 0$ and $e_{F} \equiv 0$.

In contrast to Shaw and Schumann [47], we do not explicitly prescribe a drag law with a certain drag coefficient. Knowledge about the drag coefficient is not a prerequisite for the simulations here. Instead, we add these repelling forces at all grid cells filled with tree elements. In contrast to the RANS simulations as conducted by [23] the velocity is not prescribed to a certain value in the tree cells and the repelling forces are calculated implicitly. The relaxation terms attenuate the flow to the given states within a time comparable to one time step, i.e. $\beta^{-1} \sim \Delta t$ [53].

A new facet of the simulations presented in this paper are IMB elements (in our case, the crown space of the virtual forest) whose temperature is prescribed by $\theta_{F}=\theta_{e}+\Delta T$ in (3). Recently, the IMB method was employed successfully in EULAG to model neutrally and stably stratified air flow past buildings [53], as well as ground water flow through porous media [54]. Here, we aspire an extension: we model seamlessly over a wide range of scales and Reynolds numbers, from the individual porous fractal trees and branches to a scale, that is comparable to urban canopy flow.

In EULAG, the prognostic equations (2)-(4) are cast in generalized curvilinear, time-dependent coordinates $[27,38]$. Here, all conducted calculations used the Cartesian coordinates with a stretched vertical coordinate exclusively $[48,60]$. The PDEs (2)-(4) are integrated numerically using a second-order-accurate, semi-implicit, non-oscillatory forward-in-time (NFT) method. This particular numerical approach, its derivation, implementation, and diverse applications are described in [49-54].

The robustness of the solver in EULAG allows to represent the forest canopy from the diameter of individual branches to the height of the trees. Thereby, the IMBs are treated implicitly which facilitates the correct modeling of the forest canopy and simulating its response on the flow without directly prescribing a certain drag law $[53,54]$.

\subsection{Pythagoras grove: canopy modeling at plant-scale}

The self-similar ramifications of fractal tree elements can be easily computed numerically by constructing individual Pythagoras trees in the $y-z$-plane with a given fractal dimension (Fig. 1); see "Appendix B" for a brief outline of the algorithm. The solid tree elements refer to the computational grid cells where the IMB forces act. To construct a 3D tree, the fractal structure of the $y-z$-plane is duplicated in $x$-direction. 


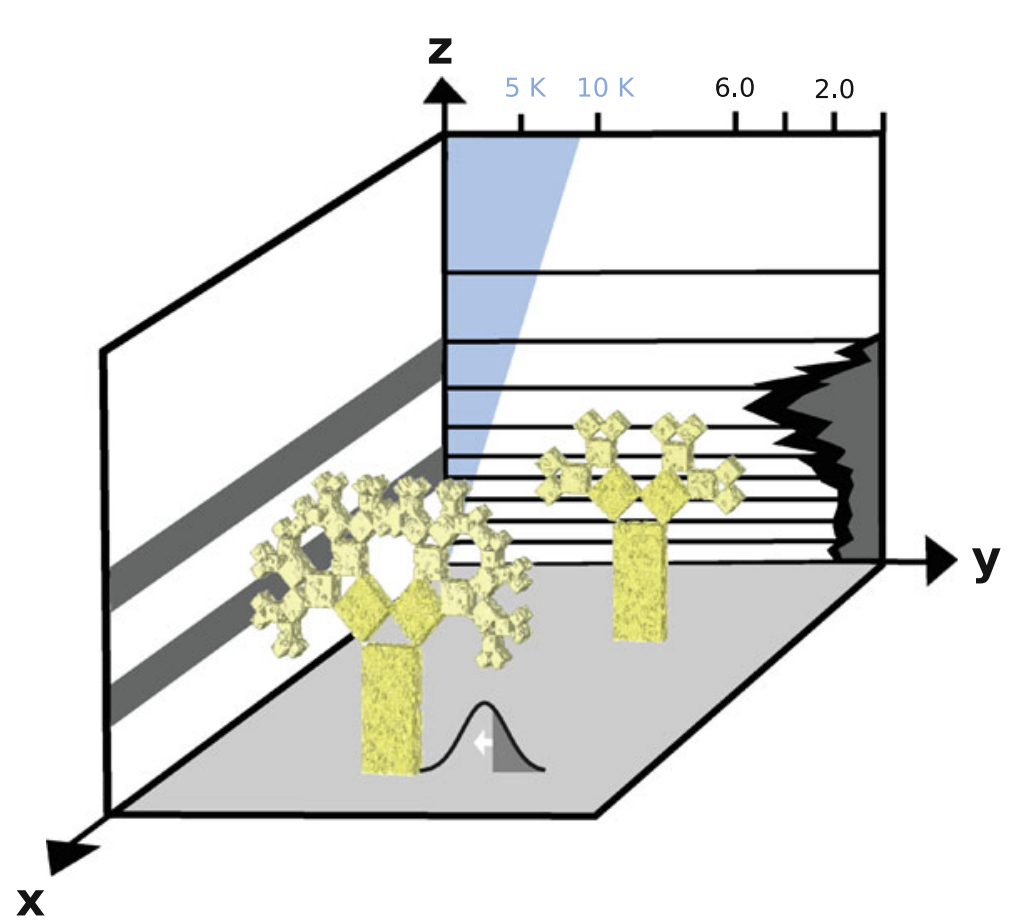

Fig. 1 Computational domain containing two illustrative fractal Pythagoras trees (enlarged). The different yellow coloring differentiates the crown space (light yellow) from the trunk space and the lower branches (yellow). For heated runs, the crown space was $\Delta T=3.35 \mathrm{~K}$ warmer than the ambient air. Height variations of tops and the crown bottoms of the sixteen virtual trees are marked by grey bars in the $x-z$-plane on the left. The leaf area density profiles $\operatorname{LAD}(z)$ are plotted for two different values of LAI $\approx 2.8$ (black) and LAI $\approx 1.9$ (grey); see text for discussion. Additionally, the vertically stretched grid is applied with an uniform stretching up to the maximum tree top is sketched in the $y-z$-plane. For the runs prescribing stable background stratification, a linear stratification was applied with an about $9.5 \mathrm{~K}$ higher temperature at model top

Each fractal tree consists of a trunk with a quadratic base area of edge length $l_{0}=0.80 \mathrm{~m}$ and a given height. The number of branch generation in the crown space is denoted by $f$ and the fractality $\|f\|_{\text {tree }}=\max (f)$ for a particular tree. For the sixteen Pythagoras trees, $\|f\|_{\text {tree }}$ varies between 5 and 8 . The length of each branch of generation $f$ can be determined by $l_{f}=l_{0} r^{f}$, where $r=1 / \sqrt{2}$ is the scaling ratio for each Pythagoras tree. The lengths of the branches cover a range from $l_{1}=0.57 \mathrm{~m}$ down to $l_{8}=0.05 \mathrm{~m}$.

To create the cluster of fractal trees, random numbers are drawn from a Gaussian distribution to determine the individual extent (maximum tree top height is $h=9.5 \mathrm{~m}$ ) and fractality $\|f\|_{\text {tree }}$ for each tree. Also the $x-y$ positions are varied by a Gaussian distribution with a standard deviation $\sigma_{y}=1.0 \mathrm{~m}$. To generate a virtual forest canopy, we distribute a total number of sixteen different Pythagoras trees in the computational domain in a way that the branches of the different trees do not overlap each other. A typical length scale for the size of the gaps, i.e. the distance between the individual tree crowns, in $y$-direction is $\approx 10 \mathrm{~m}$ and in $x$-direction it is $1.6 \mathrm{~m}$.

To allow for additional randomness of the forest flow, each tree is perforated according to a Gaussian distribution. Thereby, the Gaussian distribution can be symmetric or the likelihood of holes can be increased, if the distribution is shifted to the right (bell-shaped curve, Fig. 1). In this way, the LAD becomes scaledependent and the porosity of the tree increases towards the outer branches. The random distribution of holes in the solid tree represents realistic features such as leaves or broken branches in the crown space, that cannot be resolved explicitly. The porous trunks act as a hydrodynamically rough surfaces, as does the bark of a tree in nature.

Altogether, the cluster of these fractal trees represents a porous medium experiencing the flow at different scales. Thereby, the horizontal mean of the filled cells over all trees shows a leaf area density profile $\operatorname{LAD}(z)$ of our fractal forest whose rough shape (black and grey shaded profiles in Fig. 1) resembles LAD-profiles as observed in real forest stands (personal communication Schlegel [42], MetStroem 2011). The resulting LAI is about 2.8; as listed in Table 1. This value is in the range of a LAI $\in[2,5]$, used in field-scale simulations as $[12,47]$. When the porosity increases towards the outer branches, an overall lower LAI $\approx 1.9$ results. This value agrees well to a LAI $\approx 1.6$ estimated in forest canopies during partial leaf fall as observed in [46], p. 399. 
Table 1 Typical parameters for the five different numerical computations; $N$ is the buoyancy frequency, $\Delta T$ the crown space temperature difference to the ambient air, $U_{0}$ the bulk horizontal velocity, LAI the leaf area index

\begin{tabular}{llllll}
\hline Run & Stratification & $\mathrm{N} / \mathrm{s}^{-1}$ & $\Delta T / \mathrm{K}$ & $U_{0} / \mathrm{ms}^{-1}$ & $\mathrm{LAI}$ \\
\hline 1$)$ & Neutral & 0 & 0 & 2.8 & 2.8 \\
$2)$ & Neutral & 0 & 3.35 & 2.8 & 2.8 \\
$3)$ & Neutral & 0 & 3.35 & 2.8 & 1.9 \\
$4)$ & Stable & 0.0535 & 0 & 2.8 & 2.8 \\
$5)$ & Stable & 0.0535 & 3.35 & 2.8 & 2.8 \\
\hline
\end{tabular}

\subsection{Computational Setup}

The computational domain of $\left(L_{x}, L_{y}, L_{z}\right)=(19.15 \mathrm{~m}, 19.15 \mathrm{~m}, 108.5 \mathrm{~m}) \approx(2 h, 2 h, 11 h)$ is resolved with $n=m=l=384$ grid points and periodic boundary conditions are applied in $x$ - and $y$-directions, respectively. Parallel computations using a two-dimensional domain decomposition in EULAG scale very well if the number of processors NPE $=\sqrt{n \times m}[36,39]$. Therefore, for all our computations NPE $=384$ are requested on six IBM Power6 compute nodes. The spatial resolutions are uniform in both horizontal directions and amount to $\Delta x=\Delta y=0.05 \mathrm{~m}$. In the vertical direction, a stretched grid is computed using a modified $\operatorname{arctanh}(\mathrm{z})$ as coordinate transformation for the generalized coordinates in EULAG [60]. The vertical resolution amounts to $\Delta z \approx 0.12 \mathrm{~m}$ in the trunk space, $\Delta z \approx 0.09 \mathrm{~m}$ in the crown space and up to $z \approx 3 h$, and attains $\Delta z \approx 24 \mathrm{~m}$ at the domain top. The grid increments are nearly uniform throughout the shear layer usually located above the forest canopy. More than half of the grid points are distributed in this layer. In this way, the whole surface layer with a depth of about $100 \mathrm{~m}$ can be covered while individual tree elements down to small branches are resolved in the crown space.

The initial conditions for the velocity components are $(u, v, w)=\left(U_{0}, 0,0\right)$, whereas the potential temperature fluctuations $\theta^{\prime}$, and the SGS turbulent kinetic energy $e$ are set to zero. To maintain a constant mass flux through the domain, the mean streamwise velocity over a $y-z$-plane above the flat surface is calculated. At each time step, a pressure gradient in the $x$-direction is determined by the difference of this value to $U_{0}$ divided by $\Delta t$. This auxiliary forcing is added at each grid point.

Finite differencing requires a small time step for the high-resolution numerical simulations. To achieve a Courant number $<1$, a constant time step of $\Delta t=0.002 \mathrm{~s}$ was used for each simulation. This leads to timeconsuming simulations with $n t=180,000$ integration steps for a simulation time of $360 \mathrm{~s}$ for each run. The total wall clock time for one simulation was $\approx 120 \mathrm{~h}$.

Five different simulations were performed; see Table 1 for a summary. Three runs were conducted with a neutral background stratification $N=0$ and two with a stable stratification $N=0.0535 \mathrm{~s}^{-1}$ resulting in a $\approx 9.5 \mathrm{~K}$ higher potential temperature $\theta_{e}$ at the top of the domain. To study the impact of a relatively warm tree crown, the outer tree branches were forced to be $\Delta T=3.35 \mathrm{~K}$ warmer than the ambient air $\theta_{e}(z)$ in runs 2 and 3 for initially neutral stratification and in run 5 with initially stable background stratification. A special numerical simulation (run 3 in Table 1) is devoted to study the effect of the scale-dependent LAD and a lower LAI on the flow structure.

\subsection{Analysis}

The turbulence structure of the forest flow is computed online at every time step during the simulations. The whole simulation was divided into 18 parts, each $20 \mathrm{~s}$ long. Therefore, different kind of time and space averages were calculated. In each sub-simulation starting at $t^{\text {start }}=60,80, \ldots, 340$ s mean values and turbulent moments are calculated as temporal averages at each grid point. For this purpose, the algorithm presented in "Appendix C" was applied. The temporal averages over $\Delta \tau=10$ and $20 \mathrm{~s}$ intervals are denoted by \langle\rangle$^{\Delta \tau}\left(\mathbf{x}, t_{0}\right)$ and are available at the discrete times $t_{0}=70,80,90, \ldots, 360 \mathrm{~s}$ for $\Delta \tau=10 \mathrm{~s}$ and at $t_{0}=80,100,120, \ldots$, $360 \mathrm{~s}$ for $\Delta \tau=20 \mathrm{~s}$, respectively. During the nearly stationary phase of the simulations, these local, temporal averages can be considered as a kind of ensemble mean over $\Delta \tau / \Delta t=5,000$ or 10,000 members. From these averages, vertical profiles are calculated as horizontal averages \langle\rangle$_{x y}^{\Delta \tau}\left(z, t_{0}\right)$. Long-term mean values denoted by \langle\rangle$_{t}(x, y, z)$ were computed over a period of $300 \mathrm{~s}$. From these mean values, horizontal means are calculated at each $z$-level to yield vertical profiles \langle\rangle$_{x y t}(z)$, briefly denoted as \langle\rangle$(z)$.

In addition, selected diagnostic quantities were calculated online. For example, a quadrant analysis of the vertical momentum flux $\left\langle u^{\prime} w^{\prime}\right\rangle$ according to the sign of $u^{\prime}$ and $w^{\prime}$ was employed to calculate the ratio $\left\langle r_{u w}\right\rangle^{\Delta \tau}$ 
of $\left\langle u^{\prime} w^{\prime}\right\rangle^{\mathrm{IV}}$ to $\left\langle u^{\prime} w^{\prime}\right\rangle^{\mathrm{II}}$ which determines the relative portion of ejection and sweep events [25]. Ejections stand for upward momentum transport: $w^{\prime}>0$ and $u^{\prime}<0$ (forth quadrant), whereas sweeps depict downward transport of momentum: $w^{\prime}<0$ and $u^{\prime}>0$ (second quadrant). Both processes characterize the evolution of the internal boundary layer (IBL) above the forest canopy.

To estimate the depth of the IBL, different measures are used. First, we estimate $h_{\mathrm{IBL}}$ as the level above the canopy that is insensitive to perturbations from below, similarly to the approach in $[4,12]$ :

$$
\frac{\partial\langle u\rangle_{x y}^{\Delta \tau}(z, t)}{\partial z}=\frac{\partial\langle u\rangle(z)}{\partial z}, \quad \forall z=h_{\mathrm{IBL}}(t)
$$

Furthermore, a shear length scale $L_{s}$ defined as

$$
L_{s}=\left.\frac{\langle u\rangle}{\mathrm{d}\langle u\rangle / \mathrm{d} z}\right|_{z=h}
$$

is computed to determine the depth of the mixing layer above the canopy top; c.f. Finnigan [15], p. 526. A further measure for the IBL height used in our analysis is the vorticity thickness

$$
\delta_{w}=\frac{1}{2} \frac{\Delta U}{\|\mathrm{~d}\langle u\rangle / \mathrm{d} z\|_{\max }},
$$

where $\Delta U$ is the velocity difference across the mixing layer and $\|\mathrm{d}\langle u\rangle / \mathrm{d} z\|_{\max }$ the maximum value of the vertical gradient of the streamwise velocity inside the mixing layer; see Fig. 10 in [15] for an illustration.

Additionally, the $\lambda_{2}$-method is used as flow diagnostic to characterize the core of vortices inside the IBL and especially, inside the forest canopy [24]. We calculate the real eigenvalues $\lambda_{1} \geq \lambda_{2} \geq \lambda_{3}$ of the symmetric tensor $\mathcal{S}^{2}+\Omega^{2}$ offline, where the symmetric and antisymmetric parts of the velocity gradient tensor are denoted by $\mathcal{S}$ and $\Omega$. As mentioned in [24], this method accurately identifies the vortex core at points where $\lambda_{2}<0$ for flow situations where the vortex geometry is intuitively clear.

\section{Results for the neutral reference run}

In this section, we demonstrate the feasibility of the neutral reference simulation (run 1, see Table 1) to reproduce previously published results from field-scale LESs [12,17,47] and laboratory experiments as [6]. Plotting vertical profiles $\langle u\rangle_{x y}^{20 \mathrm{~s}}$ and the ratio between ejection and sweep events $\left\langle r_{u w}\right\rangle_{x y}^{20 \mathrm{~s}}$ as functions of time results in so-called Hovmoeller diagrams which illustrate the overall flow evolution (Fig. 2). To facilitate a quantitative comparison, vertical profiles of mean velocities $\langle u\rangle$, variances of the streamwise velocity $\sigma_{u}^{2}=\left\langle u^{\prime} u^{\prime}\right\rangle$, the vertical momentum flux $\left\langle u^{\prime} w^{\prime}\right\rangle$, and $\left\langle r_{u w}\right\rangle$ were computed and contrasted with published results (Fig. 3).

The temporal evolution of the streamwise velocity $\langle u\rangle_{x y}^{20 \mathrm{~s}}$ is dominated by the formation of a gradually ascending shear layer above the forest canopy. Additionally, a velocity maximum develops inside the trunk space for $t<150 \mathrm{~s}$ (Fig. 2a). Later, this sub-canopy jet vanishes due to turbulent mixing in the surface layer, until it disappears completely after $300 \mathrm{~s}$. A wind jet in the sparse sub-canopy layer was already simulated in a former field-scale study under neutral atmospheric conditions; c.f. p. 103, Fig. 5a in [12].

The normalized streamwise velocity profile $\langle u\rangle(z) / U_{0}$ as plotted in Fig. 3a resembles a typical plane mixing layer profile, where two co-flowing fluids with different velocities merge [15]. Here, $\langle u\rangle(z) / U_{0}$ is lowest inside the crown area and increases monotonically in a shear layer up to a constant free stream velocity of $\langle u\rangle(z) / U_{0} \approx 1.2$ at $z \approx 3 \mathrm{~h}$. Two inflection points are marked in Fig. 3a, where the upper point marks the transition from the low speed flow in the forest to the free flow above. The lower one indicates a second, weaker mixing layer below the crowns as the velocity in the trunk space increases slightly.

The mean velocity profile $\langle u\rangle$ (Fig. 3a) agrees qualitatively with the simulations of Shaw and Schumann for $\mathrm{LAI}=2$; c.f. Fig. 2 in [47]. Additionally, the Hovmoeller diagram of $\langle u\rangle_{x y}^{20 s}(z, t)$ as shown in Fig. 2a is very similar to $x-z$ slices of time- and $y$-averaged streamwise velocity for the flow over a forest edge; c.f. Fig. 3a in [12]. There, the downstream growth of the IBL above the forest translates into a gradually expanding IBL in time for our simulation as depicted in Fig. 2a. At $t=300 \mathrm{~s}$, the IBL depth $h_{\mathrm{IBL}}$ is about $2 h$ according to Eq. 5; see the light blue line in Fig. 2a. The shear length scale above the canopy is $L_{s} \approx 0.4 h$ using Eq. 6. This value corresponds quantitatively very well to $L_{s}=0.38 \mathrm{~h}$ as deduced from the numerical simulation by [12], p. 104. We yield $\delta_{w} \approx 1 \mathrm{~h}$ for the vorticity thickness (Eq. 7), using the long-term average of $\langle u\rangle$ (Fig. 3a). 

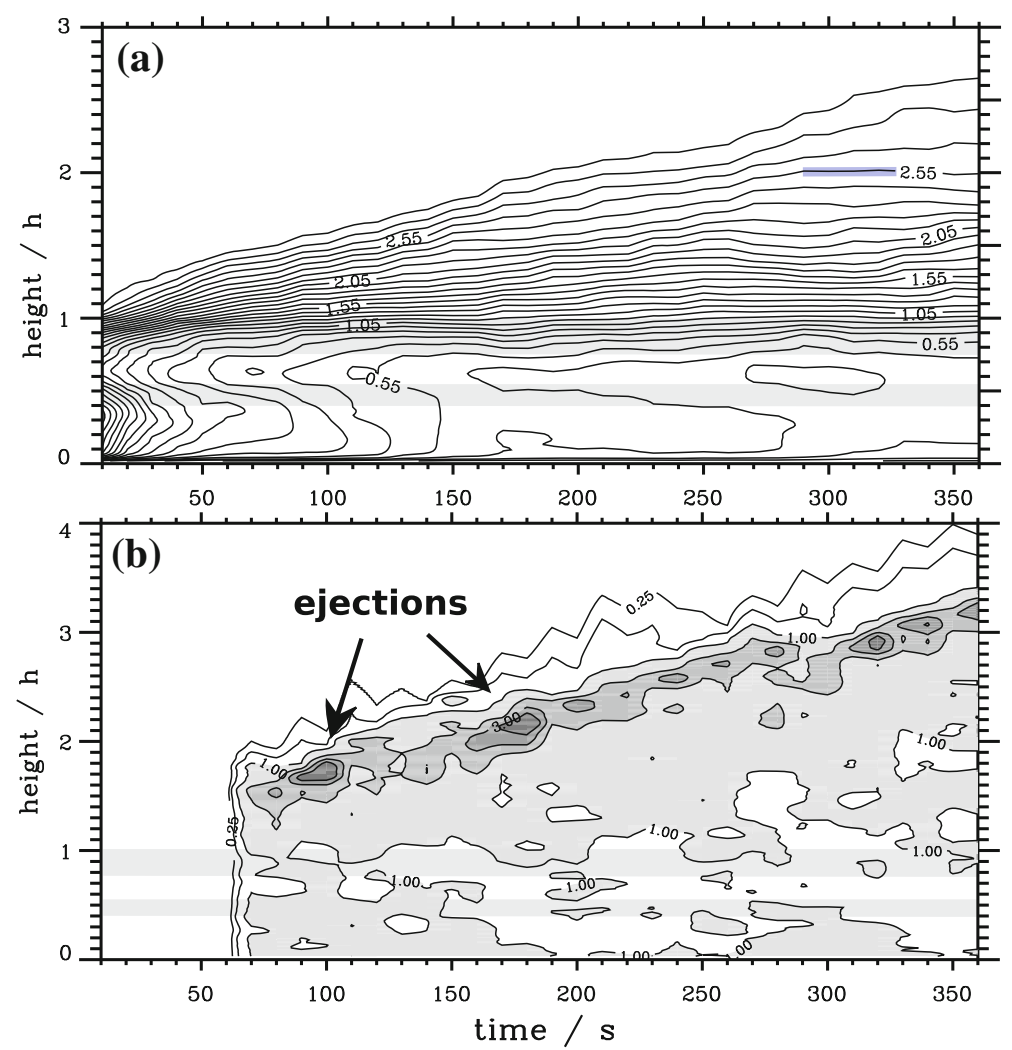

Fig. 2 Evolution of the internal boundary layer (IBL) as depicted by Hovmoeller diagrams of the mean quantities: a horizontal wind speed $\langle u\rangle_{x y}^{20 \mathrm{~s}}$ with an approximate IBL height (light blue line) of $h_{\mathrm{IBL}} \approx 2 h$ at $t=300 \mathrm{~s}$ according to Eq. 5 . b ratio $\left\langle r_{u w}\right\rangle_{x y}^{20 \mathrm{~s}}$ between ejection and sweep events. The grey shaded areas indicate dominating ejection events. The horizontal grey bars in the panels denote the variations in tree top and crown bottom heights, respectively
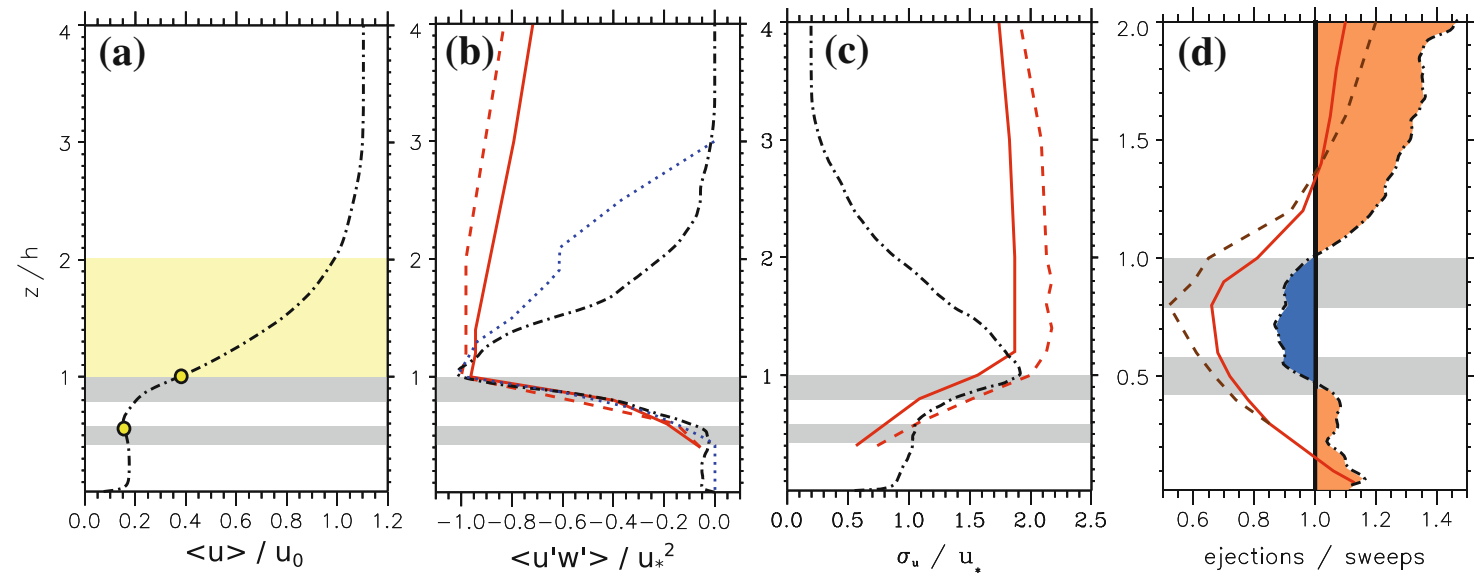

Fig. 3 Vertical profiles for mean quantities and turbulent moments for the neutral reference run (black line): a mean horizontal velocity $\langle u\rangle / U_{0}$ with inflection points as marked in yellow, b momentum transport $\left\langle u^{\prime} w^{\prime}\right\rangle / u_{*}^{2}$, c variance $\sigma_{u} / u_{*}, \mathbf{d}$ ejections/sweeps ratio $\left\langle r_{u w}\right\rangle$. Blue/orange shaded areas mark dominating ejection/sweep events. The horizontal grey bars in the panels denote the variations in tree top and crown bottom height, respectively. We compare our results to the laboratory experiments by Brunet (dashed red line), the LES by Finnigan et al. [17] (solid red line), the LES by Shaw and Schumann [47] (dotted blue line), and the wind tunnel studies by Raupach (dashed brown line) [41]

The growth of the IBL can be explained physically by the two modes of momentum transfer, i.e. by ejections and sweeps produced in the shear layers. The Hovmoeller diagram in Fig. $2 b$ shows the evolution of the ratio of ejection to sweep events $\left\langle r_{u w}\right\rangle_{x y}^{20 \mathrm{~s}}$. At the top of the growing IBL, ejection events dominate over sweeps. 
These ejection events appear discontinuously in time and show a duration of up to $30 \mathrm{~s}$. The interval between the ejection events ranges between 60 and $80 \mathrm{~s}$. Below the top of the IBL, sweep motions are simulated and dominate inside the crown space after $150 \mathrm{~s}$. Near the ground, momentum transfer by sweeps and ejections occurs with similar intensity, i.e. $\left\langle r_{u w}\right\rangle_{x y}^{20 \mathrm{~s}} \approx 1$.

Interestingly, a kind of symmetry is observed in the long-term averaged $\left\langle r_{u w}\right\rangle$-profile at the height of the inflection points of the $\langle u\rangle$-profile (Fig. 3d). The magnitude of sweeps and ejections balances, i.e. $\left\langle r_{u w}\right\rangle=1$, at the canopy top (upper inflection point) and at the bottom of the crown space (lower inflection point). Inside the crown space, sweeps dominate, whereas ejections dominate at the ground and above the forest canopy. Thus, horizontal momentum is scattered/collected at the upper/lower inflection point in the mean.

The simulated balance between sweep and ejection modes at the canopy top in our reference simulation is in agreement with former field-scale simulations [12,15]. Furthermore, the range of $\left\langle r_{u w}\right\rangle_{x y}^{20 s}$-values inside the IBL agrees quantitatively with the results of laboratory experiments and former LES; c.f. Fig. 3d with vertical profiles in Fig. 2 [17]. Also the shape of the vertical $\left\langle r_{u w}\right\rangle$-profiles inside the crown space and up to the height of the IBL agrees with the former simulations. Absolute values are slightly higher in our simulations: sweeps still dominate inside the crown space, while ejections clearly dominate above the canopy top, as also observed in the forest canopy LES over a forest edge; c.f. Fig. 3e in [12]. While certain events in our fractal forest flow at the canopy top reach a value of $\left\langle r_{u w}\right\rangle_{x y}^{20 s}\left(z, t_{0}\right) \in[2.0,3.0]$, maximum averaged $r_{u w}$-values range between 1.8 and 2.0 in their forest edge flow simulation [12].

The magnitude of the normalized vertical momentum flux $\left|\left\langle u^{\prime} w^{\prime}\right\rangle\right| / u_{*}^{2}$ reaches a maximum at the top of the canopy (Fig. 3b). As expected for a mixing layer profile, $\left|\left\langle u^{\prime} w^{\prime}\right\rangle\right|$ decreases monotonically with height until it approaches 0 above $z \approx 3 h$. Inside the crown space, the magnitude of the vertical momentum flux decreases almost linearly to a value below $0.1 u_{*}^{2}$ at crown bottom height. In the trunk space, the momentum flux slightly increases and approaches zero at the ground. Our momentum flux profile (Fig. 3b) agrees quantitatively very well with former field-scale simulations and laboratory experiments within the forest. However, and due to different boundary conditions applied in our simulations the momentum flux declines quicker to zero above the forest; compare Fig. 1c in [17] with Fig. 3b. Furthermore, the upstream conditions differ: in the laboratory experiments, an extra rough area of gravel and a fence at the height of the canopy was installed to disturb the flow, before it approaches the canopy [6]. However, in the altitude range above the forest canopy our results agree with the simulation of Shaw and Schumann [47], who used the same method to force a constant mass flux through the computational domain. Our momentum flux profile approaches zero at a height, where the vertical velocity profile approaches the free stream velocity, exactly as in [47].

The normalized horizontal velocity variance $\sigma_{u} / u_{*}$ is nearly uniform in the sub-canopy space with $\sigma_{u} / u_{*} \approx 1.0$ at the bottom of the crown. It increases almost linearly inside the crown space, before it reaches its maximum of $\sigma_{u} / u_{*} \approx 2.0$ at the canopy top (Fig. 3c). Aloft, it decreases monotonically up to a height of $z \approx 3.3 \mathrm{~h}$, where it approaches a constant value of $\sigma_{u} / u_{*} \approx 0.2$. The slope of the profile inside the crown space is again the same as for wind tunnel studies [6] and the field-scale LES; c.f. Fig. 1b in [17]. Furthermore, the normalized values agree very well with the wind tunnel experiment of waving wheat by [6] inside the crown space; c.f. Fig. 4 in [6]. In the trunk space, the profile approaches a constant value as in [17].

A special feature of our numerical simulations are ubiquitous small-scale flow structures inside the canopy layer produced by the flow around the fractal trees. These resolved flow structures are the result of our numerical approach to represent the forest canopy as being composed of separated, numerically resolved solid obstacles. For example, intense shear layers (magnitude of the 3D vorticity $|\eta| / U_{0}>5 \times 10^{-3} \mathrm{~m}^{-1}$ ) develop around the aerodynamically rough trunks and branches of the fractal trees as shown for the neutral reference run in Fig. 4a. The shear layer which develops as response to the flow through the ensemble of forest elements is evident as tattered green band spanning laterally above the tree in Fig. 4a. The shear layer varies in thickness and intensity, depending on the local position. However, the estimated value of $L_{s} \approx 0.4 h$ fits very well to the mean depth of the shear layer.

In the locally unsteady flow, vortices are generated in the lee of the trunks. In the course of the simulation these coherent structures detach and are advected downstream (Fig. 4b). Wake vortices can have a crucial impact on the mean flow and on the associated transport processes; e.g. TKE production [3]. They are also the means of the upward $\mathrm{CO}_{2}$ transport from the ground of the forest canopy [8]. Their characteristic length and time scales were determined by recent observations in a field campaign $[8,13]$. Their effect on the energy spectrum was discussed in [15] and illustrated with Fig. 2 in [8]. Vortex shedding is a typical example for a downscale energy cascade process. In the wake of the trees the shear layer deforms to a wake vortex band, that gets twisted and dissipates finally. This cycle can be observed several times at a number of positions inside the fractal forest; exemplified by two $\lambda_{2}$-snapshots in Fig. $4 \mathrm{~b}$. 

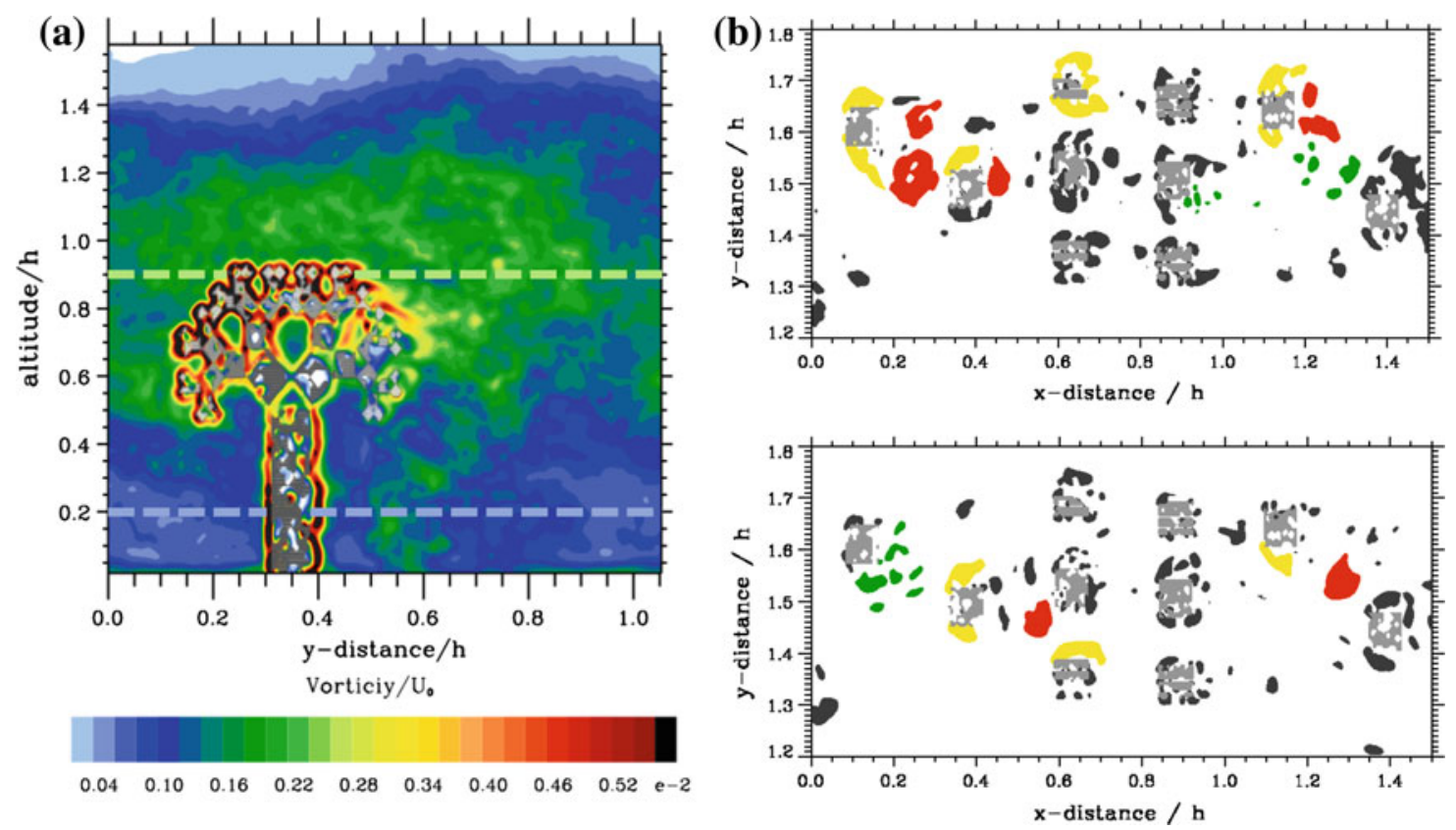

Fig. 4 a Absolute value of the vorticity vector $|\eta| / U_{0}\left(\mathrm{~m}^{-1}\right.$, color shaded $)$. The green and blue thick dashed lines indicate the heights, where power spectra were calculated (Fig. 10). b $\lambda_{2}$-snapshots of the flow at a height of $z=0.4 h$ and at two different times: $t=120 \mathrm{~s}$ (above) and $t=240 \mathrm{~s}$ (below). Shear layers are marked by yellow, wake vortices behind the trunks by red, and, finally, dissipative structures by green; black is unidentified. The different stages of vortex shedding and decay were determined intuitively by human eye. In $\mathbf{a}, \mathbf{b}$ the gray-shaded areas depict trunks of fractal trees

\section{Diabatic effects on the canopy flow}

Under neutral conditions, the potential temperature $\theta$ is constant and vertical motions cannot produce hydrostatic pressure anomalies. These pressure anomalies are generated by heating the crown space of the trees diabatically or by vertical motions in a statically stable background stratification. Under stable atmospheric background conditions, $\theta(z)$ increases with height. Without diabatic heating, lower $\theta$-air is transported upward from below mechanically and, consequently, a cold anomaly must be produced. A cold anomaly aloft will produce a high pressure anomaly below which results in horizontal pressure gradients which accelerate/decelerate and deflect the wind. In this chapter, we investigate the impacts of a stable background stratification and the local heating of the tree crowns on the flow.

First, we first illustrate the evolution of the thermal stratifications as simulated in the stratified runs. Runs 2 and 3 have initially neutral stratification, whereas runs 4 and 5 are initially stably stratified. All stratified runs, except run 4 have heated tree crowns (Table 1). We show three exemplary Hovmoeller diagrams of mean potential temperature profiles $\langle\theta\rangle_{x y}^{20 \mathrm{~s}}(z, t)$ in Fig. 5. Then, the formation of the IBL is studied by considering the mean horizontal velocity $\langle u\rangle(z)$ and the momentum flux profiles $\left\langle u^{\prime} w^{\prime}\right\rangle(z)$ for the stratified simulations (Fig. 6). Vertical heat flux profiles are depicted in Fig. 7, before the formation of temperature ramps and rising thermals are studied in detail (Fig. 8).

\subsection{Temperature field}

Figure 5 illustrates the evolution of the thermal stratification for the diabatically influenced numerical simulations. For the run without diabatic heating of the crowns and stable background stratification, turbulent mixing weakens the thermal stratification from the surface up to a height of $z \approx 2 h$ (run 4, Fig. 5a). At the end of the simulation, the potential temperature difference across this layer is about $0.4 \mathrm{~K}$ smaller than at the beginning. This is consistent with the upward transport of lower $\theta$-air above the forest canopy and the downward transport of higher $\theta$-air in the trunk space. The turbulent mixing in the IBL works counter gradient to dissolve the initial stratification (Fig. 5a). 

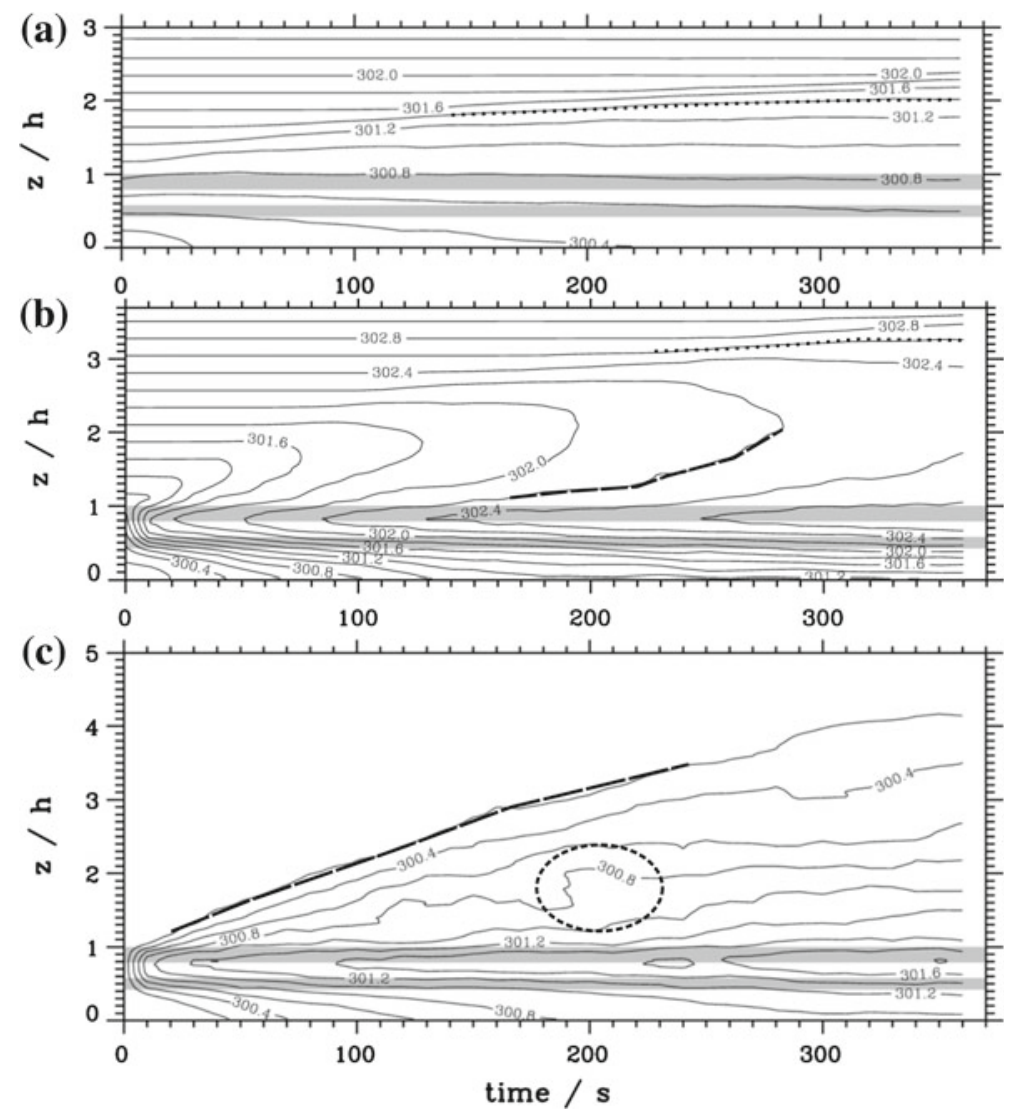

Fig. 5 Temporal evolution of mean potential temperature $\langle\theta\rangle_{x y}^{20 \mathrm{~s}}(z, t)$ (Hovmoeller diagrams) for run 4 (a; stable and not heated), run 5 (b; stable and heated), and run 2 (c; neutral and heated). The dotted line in (a) marks a temperature inversion at $z \approx 2 h$. In $\mathbf{b}$, the dotted line indicates an elevated temperature inversion at $z \approx 3 \mathrm{~h}$ after $200 \mathrm{~s}$ and the thick dashed line marks an evolving unstable stratification directly above the canopy top. In c, the dashed line marks a sudden temperature increase at $t=200 \mathrm{~s}$. The horizontal grey bars in the panels denote the variations in tree top and crown bottom heights, respectively

In contrast, the thermal stratification in the trunk space is enhanced, if the crowns are heated as depicted for the runs 2 and 5 (Fig. 5b, c), where warmer air is gradually transported downwards. For the run with stable background stratification, an average temperature difference of $\Delta \theta \approx 1.2 \mathrm{~K}$ develops during the simulation (run 5, Fig. 5b). In the run with neutral stratification, the same process generates a stable but weaker stratified layer in the trunk space (run 2, Fig. $5 \mathrm{c}$ ) and the air at crown top is on average $\approx 0.6 \mathrm{~K}$ warmer compared to the ground. The development of a stably stratified layer in the trunk space has been often observed in forest canopies in nature during the day; chapter 8.2, pp. 565-566 [15]. In our simulations, the downward heat transport is explicitly resolved and reproduces the observed effect. A quantitative comparison with field observations is planned.

Above the forest, the upward transport of high $\theta$-air diminishes either the initial stable stratification (Fig. 5a, b) or-as for the run with neutral background stratification-establishes a convective unstable stratification (Fig. 5c). In this unstable layer, a number of warm outbursts can be identified in the Hovmoeller diagram, e.g. a sudden temperature increase at $t \approx 200 \mathrm{~s}$, see the encircled area in Fig. $5 \mathrm{c}$. Common to all simulations is the gradual growth of the IBL height. Compared to the reference simulation (Fig. 2), the IBL grows faster for the runs with heated crowns and much slower for the run without heating and with stable background stratification (run 4, Fig. 5a).

\subsection{Evolution of the internal boundary layer through momentum transport}

The impacts of the ambient stratification and the diabatic heating of the tree crowns on the streamwise velocity $\langle u\rangle(z)$ and the vertical momentum flux $\left\langle u^{\prime} w^{\prime}\right\rangle(z)$ profiles are evident from Fig. 6. Higher wind speeds in the 

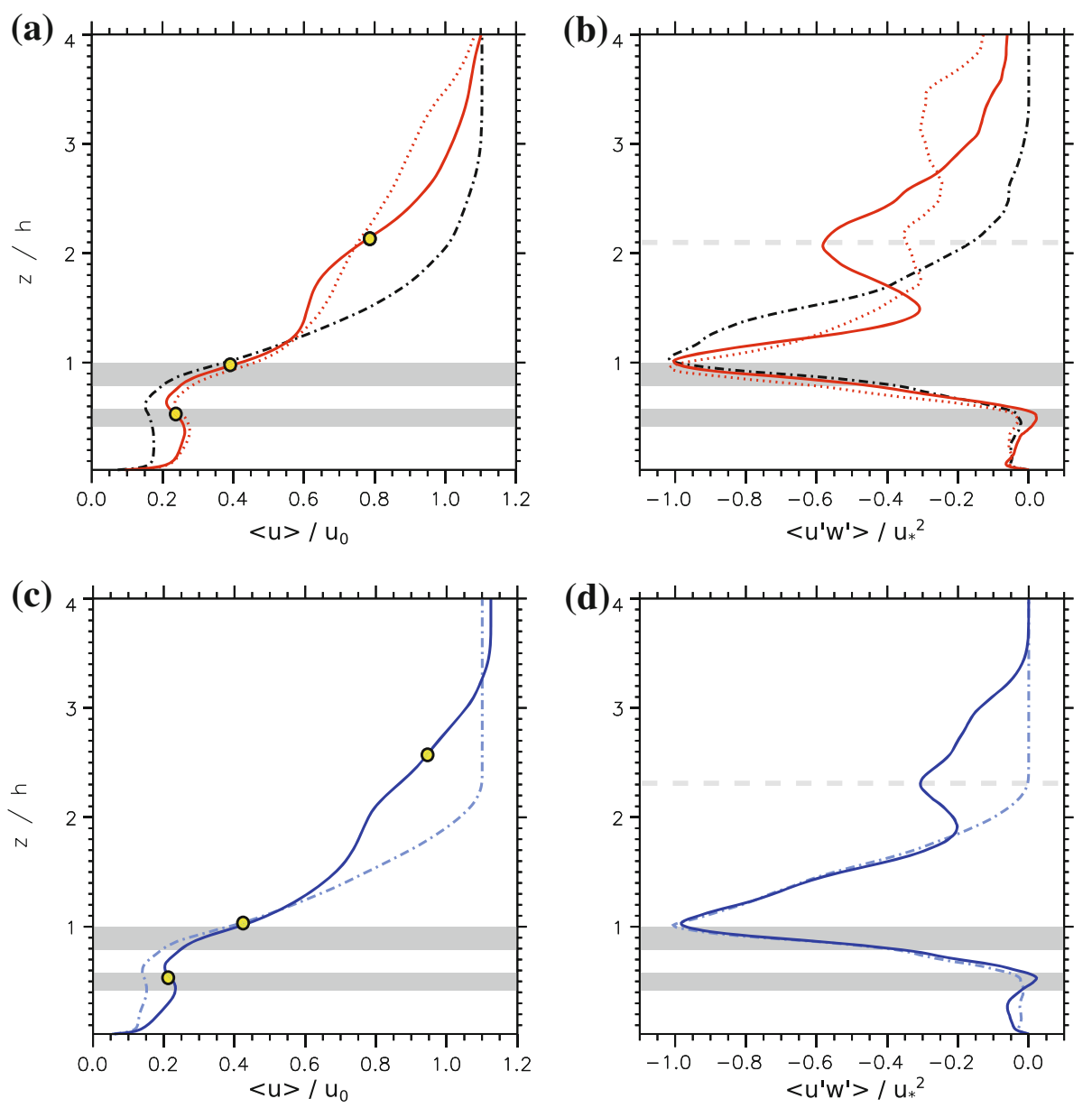

Fig. 6 Vertical profiles of the normalized mean horizontal velocity $\langle u\rangle / U_{0}$ (left panels) and the normalized vertical momentum flux $\left\langle u^{\prime} w^{\prime}\right\rangle / u_{*}^{2}$ (right panels) for simulations in a neutrally (top panels) and a stably stratified environment (bottom panels). Solid lines for runs with heated crowns: red run $2(\mathbf{a}, \mathbf{b})$, blue run $5(\mathbf{c}, \mathbf{d})$. Additionally, the simulation with the scale-dependent $\operatorname{LAD}(z)$ is marked by red dotted lines in $(\mathbf{a}, \mathbf{b})$. The dash-dotted lines in all panels refer to both simulations where the crowns are not heated, i.e. runs 1 and 4, respectively. Yellow bullets mark inflection points in the velocity profiles. Elevated shear layers are marked by a thick, horizontal dashed line at the corresponding extrema in the momentum flux profiles. The horizontal grey bars in the panels denote the variations in tree top and crown bottom heights, respectively

trunk space are simulated in the runs with diabatically heated trees for both neutrally and stably stratified conditions (Fig. 6a, c). The enhancement of the sub-canopy jet can be explained by the warm rising air aloft which draws in air from the sides, a process similar to the draft in a chimney [45]. Due to mass conservation the horizontal velocity in the trunk space must increase. As a result, the shear in $\langle u\rangle(z)$ intensifies and generates a pronounced inflection point. Eventually, instabilities inducing mechanical turbulence, stronger mixing and higher momentum transport are triggered at the crown bottom.

Inside the forest canopy and directly above, the shape of the normalized momentum flux profiles $\left\langle u^{\prime} w^{\prime}\right\rangle(z)$ for the runs with stable background stratification (Fig. 6b, d) is similar to that for the reference run 1 (Fig. 3b). Inside the canopy, the magnitude of the momentum flux $\left|\left\langle u^{\prime} w^{\prime}\right\rangle\right| / u_{*}^{2}$ increases with height and the momentum transport always maximizes at its top. To quantify the momentum transport at canopy top, we calculate the friction velocity as $u_{*}=\left.\left(-\left\langle u^{\prime} w^{\prime}\right\rangle\right)^{1 / 2}\right|_{z=h}$ in accordance with [17]. Table 2 lists the results for the five runs. Compared to the runs without heated crowns, the friction velocity $u_{*}$ is slightly enhanced when the crowns are warmer than the ambient air: $u_{*}=0.21 \mathrm{~ms}^{-1}$ for run 3 and $u_{*}=0.19 \mathrm{~ms}^{-1}$ or run 5 ; c.f. Table 2 . The simulated friction velocities at canopy top agree quantitatively with former field experiments, where observed $u_{*}$-values are $u_{*} \in\left[1.0 \times 10^{-2}, 1.0\right] \mathrm{ms}^{-1}$; c.f. Table II in [61].

Notably, in the runs with diabatically heated crowns elevated shear layers are produced at $z \approx 2 h$ (Fig. 6a, c) which are also visible as extrema in the corresponding $\left\langle u^{\prime} w^{\prime}\right\rangle$-profiles (Fig. 6b, d). As expected, the shear 


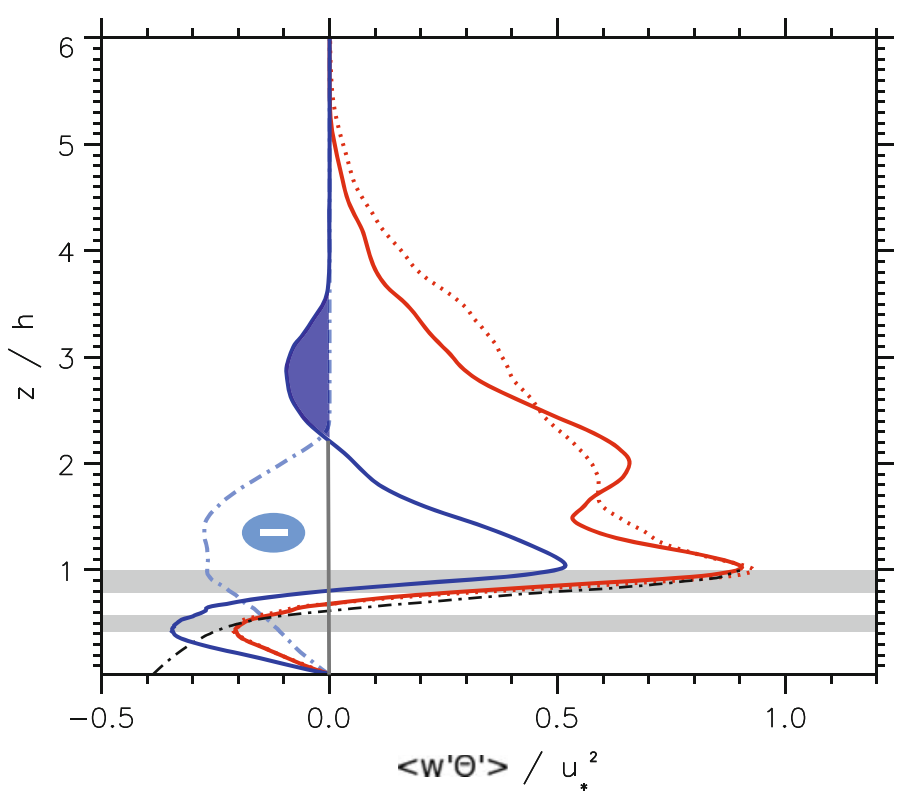

Fig. 7 Mean normalized heat flux profiles: $\left\langle w^{\prime} \theta^{\prime}\right\rangle(z)$ for run 2 (red solid line), run 3 (red dotted line), run 4 (blue dash-dotted line), and run 5 (blue solid line). For run specifications, see Table 1. For comparison, the parametric model of the heat flux (Eq. 8) with an extinction coefficient $\alpha=0.7$ is displayed by a black dash-dotted line inside the canopy space; see text for discussion

magnitude is smaller for the runs with stable background stratification (Fig. 6c) compared to both simulations with initially neutral stratification (Fig. 6a). For the special run employing a scale-dependent $\operatorname{LAD}(z)$ ), a second elevated shear layer evolves at $z \approx 3 h$ (Fig. 6a, b). A further, however, weaker mixing layer is induced by convective motions for the runs with initially neutral stratification and heating. It can be identified between $z \approx 2$ and $3 \mathrm{~h}$ for runs 2 and 3 (Fig. 6a). The formation of this elevated mixing layer increases the total height of the IBL; c.f. Fig. 10 in the review paper [15], where the height of one mixing layer increases in the course of a laboratory tank experiment. The appearance of elevated shear layers above the canopy top was also detected in other LES results of forest canopy flow under diabatic conditions (Bohrer, private communication at iLEAPS conference 2011).

The total depth of the IBL $h_{\mathrm{IBL}}$, here estimated from vorticity thickness $\delta_{w}$ (Eq. 7), evolves as sum of the individual mixing layers and is listed for the different runs in Table 2. Consequently, the deepest IBL of $h_{\mathrm{IBL}}=3 h$ was simulated for the run with heated tree crowns and a scale-dependent LAD under initially neutral stratification. The shallowest IBLs were estimated for the non-heated runs with neutral and stable background stratifications. In general, heating increases the IBL height, whereas a stable stratification decreases it. This is in agreement with findings of Stull [56], p. 104, Fig. 3.7. Furthermore, the results of our numerical simulations are in agreement with observations in [58], where the IBL height "cannot be assumed as constant but rather reflects the spatial and temporal evolution of canopy and above-canopy flow".

\subsection{Simulated thermodynamic phenomena}

Thermodynamic processes are investigated by discussing the profiles of the resolved vertical heat flux $\left\langle w^{\prime} \theta^{\prime}\right\rangle$ as depicted in Fig. 7. For all runs with heated crowns, $\left\langle w^{\prime} \theta^{\prime}\right\rangle(z)$ shows pronounced extrema at the lower and upper edges of the crown space. The down- and upward transport of warm crown air by turbulent eddies warms the trunk space and the air directly above the forest canopy because $\partial \theta / \partial t \sim-\partial\left\langle w^{\prime} \theta^{\prime}\right\rangle / \partial z>0$. In the crown space, $\left\langle w^{\prime} \theta^{\prime}\right\rangle$ increases exponentially with height, up to the canopy top, where it maximizes.

Above the canopy top, the heat flux profiles decline up to the top of the IBL, where turbulent transport ceases at $z \approx 5 h$. In this height range, the $\left\langle w^{\prime} \theta^{\prime}\right\rangle$-profiles resemble those of a convective boundary layer; c.f. Figs. 3.1-3.3, pp. 98-100 in [56]. The heat source in our simulations is the warm reservoir above the tree crowns. As expected, an entrainment zone above the mixing layer only forms in the numerical simulation with stable thermal stratification and heated crowns (blue-shaded area in Fig. 7). For both runs with heated tree crowns and initially neutral stratification, but different LAD-profiles, elevated $\left\langle w^{\prime} \theta^{\prime}\right\rangle$-maxima are simulated between 

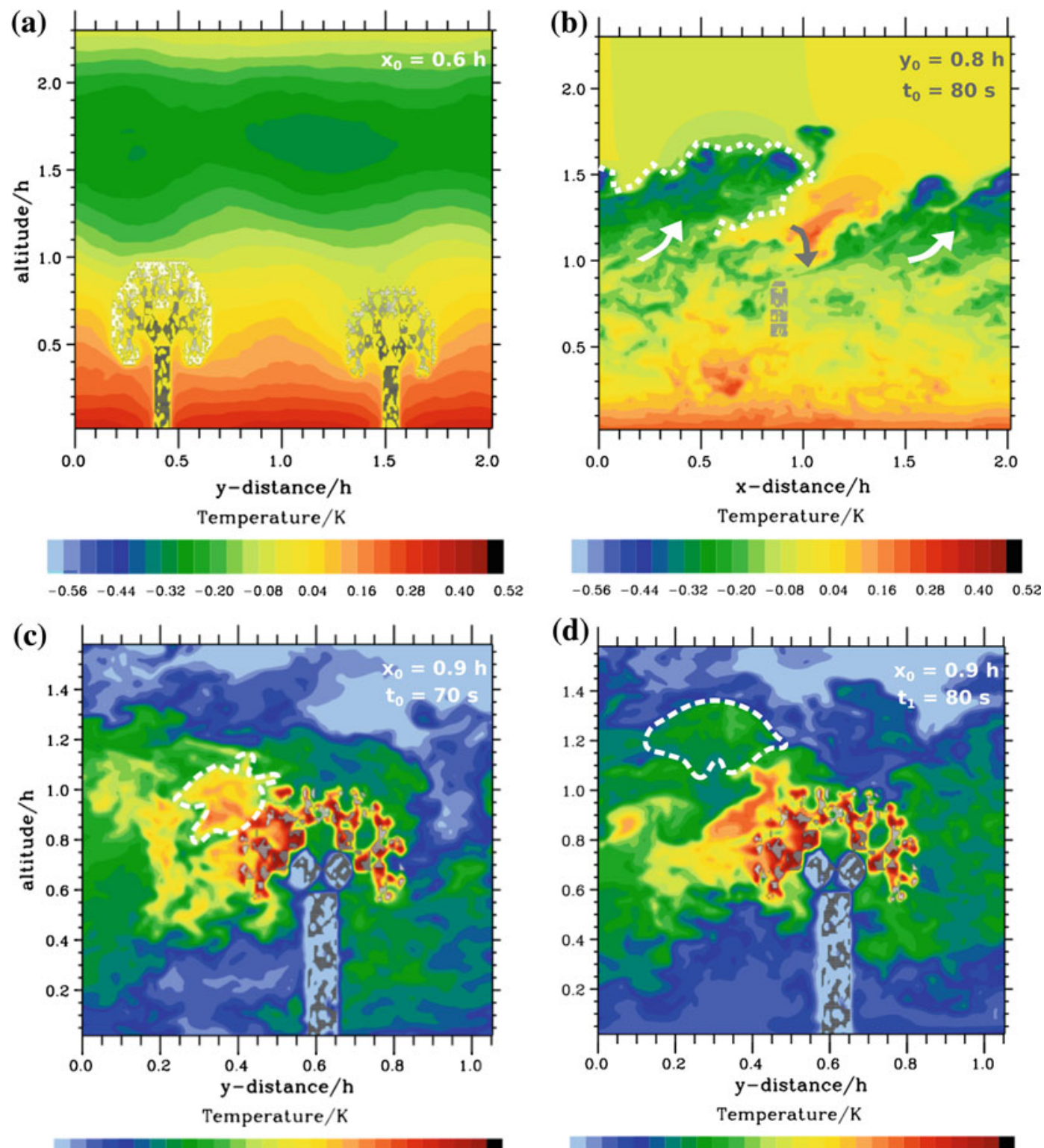

$\begin{array}{llllllllll}300.1 & 300.5 & 300.8 & 301.2 & 301.6 & 301.9 & 302.3 & 302.6 & 303.0 & 303.4\end{array}$

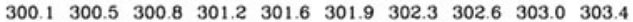

Fig. 8 Thermal structure of the forest flow under stable (run 4) and neutral (run 3) background stratification. Upper row (run 4): Potential temperature perturbation field $\left\langle\theta^{\prime}\right\rangle_{t}\left(x_{0}, y, z\right)$ as long-term mean over $300 \mathrm{~s}$ in a $y-z$ cross-section (a) and as a snapshot $\theta^{\prime}\left(x, y_{0}, z, t_{0}\right)$ at $t_{0}=80 \mathrm{~s}$ in a $x-z$ cross-section (b). Ejection and sweep events are marked with white and gray arrows, respectively. Bottom row $\theta$-snapshots around a tree as $y-z$ slice located at $x_{0} \approx 0.9 \mathrm{~h}$. In the lower row (run 3): a rising thermal is enclosed by the white dashed lines after $t_{0}=70 \mathrm{~s}(\mathbf{c})$ and $t_{1}=80 \mathrm{~s}(\mathbf{d})$ of simulation time. It was determined by inspection of animated pictures, where temperature gradients and the coherency of the pattern was visible. The gray shaded porous structures display the tree elements

$z \approx 2$ and $3 \mathrm{~h}$. Consequently, there exist layers, where the air is diabatically warmed/cooled exclusively by the turbulent transport of warmer/cooler air from above and below.

Thereby, an interplay between consumption and production of TKE and potential energy occurs. Buoyant production of TKE is simulated at the canopy top in the runs with heated tree crowns (runs 2, 3 and 5 in Table 2). Buoyant consumption occurs for run 4 with stable background stratification. Without heated crowns, the flux Richardson number $R i \sim-\left\langle w^{\prime} \theta^{\prime}\right\rangle /\left.\left\langle u^{\prime} w^{\prime}\right\rangle^{2}\right|_{z=h}=-\left\langle w^{\prime} \theta^{\prime}\right\rangle / u_{*}^{2}$ (Fig. 7) is positive and still $R i<1$ (Table 2). Thus, the flow at canopy top is dynamically unstable. 
Table 2 Normalized IBL height $h_{\mathrm{IBL}} / h$, friction velocity $u_{*}=\left.\left(-\left\langle u^{\prime} w^{\prime}\right\rangle\right)^{1 / 2}\right|_{z=h}$ and the flux Richardson number $\left.R i\right|_{z=h}$ at canopy top $h$ for the five numerical simulations

\begin{tabular}{lllllr}
\hline Run & Initial stratification & Heated crown & $h_{\mathrm{IBL}} / h$ & $u_{*} / \mathrm{m} \mathrm{s}^{-1}$ & $\mathrm{Ri}$ \\
\hline 1$)$ & Neutral & No & 0.9 & 0.16 & 0.00 \\
$2)$ & Neutral & Yes & 1.3 & 0.20 & -0.03 \\
$3)$ & Neutral & Yes & 3.0 & 0.21 & -0.03 \\
$4)$ & Stable & No & 0.9 & 0.15 & +0.01 \\
$5)$ & Stable & Yes & 1.7 & 0.19 & -0.02 \\
\hline
\end{tabular}

In the absence of diabatic heating, an interesting thermodynamic process is simulated for forest flow under stable thermal stratification (run 4). The air inside the forest stand is heated in time as $\partial\left\langle w^{\prime} \theta^{\prime}\right\rangle / \partial z<0$ across the whole canopy layer (Fig. 7). Directly at the canopy top, the vertical heat flux becomes nearly constant resulting in a nearly time-independent, uniform temperature distribution after 100 s; c.f. Fig. 5a. Above this neutral layer, between $z \approx 1.5 \mathrm{~h}$ and $2 \mathrm{~h}$, the air is cooled. The different behavior of this particular simulation can be explained by the up- and downward transport of low- $\theta$ and high- $\theta$ air in the respective layers. This kind of turbulent exchange process was also observed in field experiments by [21] and coherent updrafts of cold air above the trees were identified. So-called temperature ramps form in the initially stable environment which represent tilted coherent patterns of relatively cold air; see Fig. 1c in [21] for a time series of observational data.

The temperature ramps are visible in snapshots of the flow structure of run 4 with initially stable stratification (Fig. 8b). Colder air is transported upwards and forms coherent structures with $\theta^{\prime}<0$; see the areas enclosed with white dots in Fig. 8b. Ejection and sweep events are responsible for up- and downward transport of warmer and colder air, respectively (indicated with white and grey arrows). In the long-term mean, this leads to potential temperature perturbations $\theta^{\prime}$ of about $0.3 \mathrm{~K}$ colder directly above the tree crowns (Fig. 8a). A band of relatively cold air forms which extends from $z \approx 1 h$ to $z \approx 2 h$ in the long-term mean.

In our numerical simulations, the solar insulation is implicitly represented by prescribing the crown's temperature as $\theta_{F}=\theta_{e}+\Delta T$. This means, our approach to warm the forest air differs from those which explicitly prescribe a $z$-dependent heat flux profile (appearing as SGS forcing in the equation for $\theta$ ) throughout the forest canopy, as done in former field-scale simulations [3,47]. In those simulations a parametric model prescribes an exponential declining vertical heat flux $Q(z)$ as:

$$
Q(z)=Q(h) \exp \left(-\alpha F_{c}(z)\right)
$$

where $F_{c}(z)=\int_{z}^{h} \operatorname{LAD}(z) \mathrm{d} z$ is a downward cumulative leaf area density and $\alpha$ the extinction coefficient for solar radiation [5]. Interestingly, the resolved vertical heat flux $\left\langle w^{\prime} \theta^{\prime}\right\rangle(z)$ of our simulations with heated crowns also shows such an exponential decline (Fig. 7). Inside the crown space the resolved vertical heat flux follows even quantitatively the parametric model of Eq. 8; see black dot-dashed line in Fig. 7. To attain this agreement with the simulations under neutral stratification, the extinction coefficient for the fitting curve plotted in Fig. 7 was chosen as $\alpha=0.7$ which is nearly the same value as $\alpha=0.6$, used by Shaw and Schumann, see p. 6 in [47]. However, the curve of $Q(z)$ is shifted to the left in Fig. 7 as $Q(z)$ cannot become negative according to Eq. 8.

In this way, the vertical heat flux $\left\langle w^{\prime} \theta^{\prime}\right\rangle(z)$ and $Q(z)$ maximize at the canopy top, where $\left\langle w^{\prime} \theta^{\prime}\right\rangle / u_{*}^{2}=$ $Q(h) \approx 0.9 \mathrm{~K} \mathrm{~s} \mathrm{~m}^{-1}$. Below, $\left\langle w^{\prime} \theta^{\prime}\right\rangle / u_{*}^{2}$ declines exponentially throughout the crown space as the parametric curve $Q(z)$ does. However, the increase of $\left\langle w^{\prime} \theta^{\prime}\right\rangle / u_{*}^{2}$ cannot be captured by $Q(z)$. The heating rate $\partial \theta / \partial t \sim$ $-\partial\left\langle w^{\prime} \theta^{\prime}\right\rangle / \partial z$ in our simulation of the diabatically heated tree crowns under neutral background stratification (run 3 in Table 1) agrees quantitatively inside the crown space with results from the parametric model in the LES by [47].

Essentially, the upward transport of warmer air is an interesting example of an upscale energy cascade. Air around the fractal tree crowns is heated diabatically and merges into plumes or updrafts [45]. These updrafts are advected downstream and rise in parts as coherent thermals above the canopy top (Fig. 8c, d). These snapshots at two consecutive output times reveal that the temperature structure in the vicinity of the crowns remains very similar where coherent lumps of warm air detach regularly from the trees and rise. Above the trees the thermals loose their buoyancy due to mixing with the ambient air. The rising air generates canopy scale eddies as suggested by results from field experiments [58]. The structure of these vortices will be discussed further in the next section. 

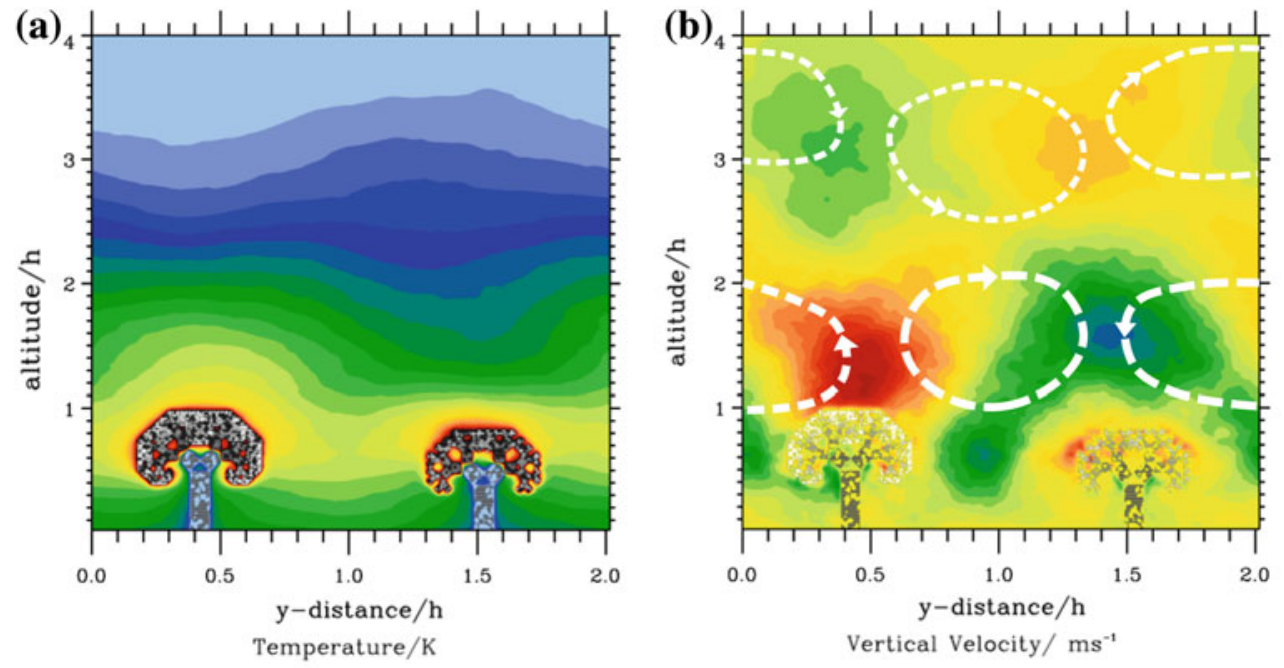

Vertical Velocity/ $\mathrm{ms}^{-1}$
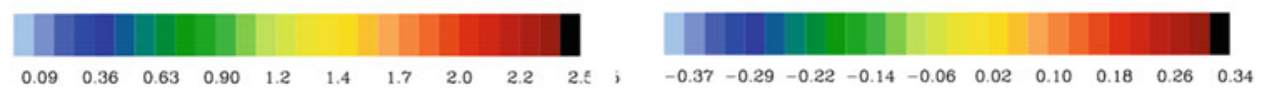

Fig. 9 Long-term averaged potential temperature perturbation $\left\langle\theta^{\prime}\right\rangle_{t}\left(x_{0}, y, z\right)$ (a) and vertical velocity $\langle w\rangle_{t}\left(x_{0}, y, z\right)(\mathbf{b})$ as $y-z-$ slice at $x_{0} \approx 0.6 \mathrm{~h}$ for the forest flow under initially neutral background stratification, with heated tree crowns (run 3 ). Average was calculated over all 150,000 time steps between $t=60$ and $360 \mathrm{~s}$. Coherent eddy motions directly above the tree crown are marked with white dashed lines

\section{Turbulence structure}

Thomas et al. [58] investigated the coherency of the flow field above a real forest stand and their wavelet analysis revealed a very high correlation coefficient between the variance spectra of the vertical wind field at different levels below $z \approx 2 h$. These observations were conducted in the late morning hours, when the atmosphere became unstable above the forest canopy; c.f. Fig. 6b in [58]. Organized, coherent flow structures above forest canopies under thermally unstable conditions were also observed in various other field campaigns $[40,46,61]$.

In our numerical simulations under neutral background stratification with heated crowns, the air becomes unstably stratified directly above the forest. Figure 9a shows long-term averaged potential temperature perturbations $\left\langle\theta^{\prime}\right\rangle$ whose magnitude decreases with height in the IBL for $z>h$. As the background temperature is constant, the total potential temperature deceases as well, i.e. $\partial\langle\theta\rangle / \partial z<0$. In this unstable environment, coherent eddy motions form which are visible as coherent up- and downdrafts inside the IBL.

Interestingly, these vortical patterns even appear in the long-term time average $\langle w\rangle_{t}\left(x_{0}, y, z\right)$ which can be considered as ensemble mean comprised of 150,000 realizations (Fig. 9b). From inspecting $\langle w\rangle_{t}\left(x_{0}, y, z\right)$, the characteristic length scales of these thermally driven vortices, their horizontal extension $Y$ and vertical extension $Z$ are determined to be about $1 h$. A characteristic scale for the vertical velocity magnitude is $W \approx 30 \mathrm{cms}^{-1}$. This leads to an eddy turnover time of $\tau \approx Z / W \approx 30$ s of these vortical motions observed in the mean vertical profile in our numerical simulation with initially neutral background stratification.

The thermal vortices visible in Fig. $9 \mathrm{~b}$ are the flow response on the evolving unstable thermal stratification directly above the canopy. A special type of instability is simulated at the elevated boundary between warm and relatively cold air (Fig. 9a): Rising thermals extending up to $\approx 2 h$ form coherent layers of warm air (Fig. 8) and the potentially colder air from above descends and intrudes into these layers. This flow type resembles a Rayleigh-Taylor instability, well-studied in the laboratory [43] or in the ocean [44]. Due to the continuous supply of warm air organized in rising thermals above the heated trees and the compensating descent, the organized and coherent vortices evolve and are maintained in the long-term average. The compensating sinking motion generates an unstably stratified $\langle\theta\rangle$-field which triggers the canopy scale eddy motion anew (Fig. 9b), a process similar to the buoyancy driven motions in a porous medium [45].

Notably, this kind of instability is also simulated in elevated layers above the forest canopy flow. It is visible in the long-term mean fields of potential temperature perturbation $\left\langle\theta^{\prime}\right\rangle_{t}\left(x_{0}, y, z\right)$ and vertical velocity $\langle w\rangle_{t}\left(x_{0}, y, z\right)$ between $z \approx 2 h$ and $4 h$. At these altitudes, potential temperature perturbations form again a wavy pattern in the $y-z$-slice with a weaker amplitude (Fig. 9a) and corresponding lower vertical velocities (Fig. 9b) than in the layer underneath. 


\section{Discussion and conclusions}

This study investigates the turbulent flow through a cluster of discrete and, as special focus, diabatically heated trees by means of high-resolution numerical simulations. For this purpose, the state-of-the-art, multiscale numerical flow solver EULAG is employed [39]. Here, the canopy was modeled by sixteen fractal Pythagoras trees whose initial crowns' temperature deviation $\Delta T$ to the environment was taken from recent field observations [33,57]. Using the IMB method, individual tree elements from the scale of small branches $(0.05 \mathrm{~m})$, the diameter of the trunks $(0.80 \mathrm{~m})$ up to the tree height of about $10 \mathrm{~m}$ were resolved in the computational domain (Fig. 1). This approach differs from published numerical methods employing a horizontally homogeneous and only vertically varying drag law to model the impact of the forest canopy on the flow at the field scale, e.g. [17,47]. Our LESs resolve both the integral flow response of the fractal Pythagoras grove-e.g. the canopy shear layer with a depth of $L_{s} \approx 0.4 h$-as well as wake vortices with a size of about $0.20 \mathrm{~m}$ generated by the interaction of the flow with the solid obstacles.

\subsection{Multiscale simulations}

The results of the reference simulation for neutral background stratification were compared with existing LES results and experimental data. Generally, our LES using individual fractal trees produces physically meaningful vertical profiles of the mean quantities and turbulent moments. The streamwise velocity component, the turbulent variances and fluxes agree with published results from field-scale simulations as $[12,17]$. The simulated momentum flux profile $\left\langle u^{\prime} w^{\prime}\right\rangle(z)$ resembles the profile obtained in a field-scale simulation inside the forest canopy quantitatively very well inside the forest; c.f. Fig. 1, p. 395 in [17]. Further aloft, the $\left\langle u^{\prime} w^{\prime}\right\rangle$-profile is similar to the profiles presented by Shaw and Schumann; c.f. Fig. 5, p. 57 in [47]. Thereby, the induced IBL in our simulation is an integral flow response solely due to the forest canopy and not due to roughness elements placed upstream of the canopy as in cited laboratory experiments of canopy flow [6].

In summary, our LES using individual fractal tress as solid obstacles are capable to simulate forest canopy flow correctly over the wide range of scales. The chosen fractal approach facilitates to bridge the gap between local, small-scale measurements, e.g. as conducted in CHATS 2007 [33], and former field-scale simulations $[12,17,47]$.

Furthermore, the numerical setup employing fractal trees is ideally suited to study multiscale processes inside the canopy by LES. Direct numerical simulations (DNS) of this type of flow are extremely expensive as the dissipation scale is very small. Dissipation of the turbulence occurs at the Kolmogorow micro-scale; see p. 563, Fig. 13.12 in [26]. Therefore, we calculate a characteristic dissipation rate: $\epsilon \approx u^{\prime 3} / \ell$. A typical fluctuation of the horizontal wind is of the order $u_{*} \approx 0.16 \mathrm{~ms}^{-1}$ for the reference run (Fig. 3). With a characteristic eddy length scale $\ell \approx h \approx 10 \mathrm{~m}$ for the largest eddies that supply energy, the dissipation rate amounts to $\epsilon \approx 4.0 \times 10^{-4} \mathrm{~m}^{2} \mathrm{~s}^{-3}$. Employing a kinematic viscosity for air of $v=1.5 \times 10^{-5} \mathrm{~m}^{2} \mathrm{~s}^{-1}$, the characteristic length scale for dissipation is: $\left(v^{3} / \epsilon\right)^{1 / 4} \approx 1.7 \mathrm{~mm}$. This resolution would be necessary for a DNS of the current flow configuration.

In our LESs, we resolve fluctuations that are $\mathcal{O}(100)$ larger than the dissipation scale. A spectral analysis of the TKE provides information at which scale energy is injected into the flow. The spectra are calculated with Fourier analysis, after the flow is fully developed and the total mean energy approaches a quasi steady state. The spectral data are averaged horizontally at three selected levels and plotted as functions of wave number $k=2 \pi / \lambda$, where $\lambda \sim \Delta x$ in Fig. 10.

The most energetic spectrum occurs inside the IBL above the forest canopy at a height of $z \approx 2.1 \mathrm{~h}$. The slope of this spectrum agrees quantitatively with the classical Kolmogorov $k^{-5 / 3}$-slope for isotropic and homogeneous flow conditions [19] for $\log k:=\log _{10} k<1.0 \mathrm{~m}^{-1}(\lambda \approx 0.62 \mathrm{~m})$. Also the spectra inside the crown and trunk space follow the $k^{-5 / 3}$ - slope, however, the energy content at these wave numbers is up to one order of magnitude lower. At smaller scales $\log k>1.4 \mathrm{~m}^{-1}(\lambda<0.25 \mathrm{~m})$ the spectra at the lower levels carry more energy due to the generation of turbulent eddies at the solid obstacles in the trunk and crown space.

In the fractal forest, coherent vortices occur at given scales in the wakes as depicted in Fig. 4; e.g. produced by leaves, stems and branches $[8,13]$. The branches in our simulation have a typical size of e.g. $l_{4}=0.20 \mathrm{~m}$ for the fourth branch generation of the Pythagoras trees. This corresponds very well to the decadic logarithm $\log k=1.5 \mathrm{~m}^{-1}$ in the spectrum and the corresponding scale of the eddy of $\lambda \approx 0.20 \mathrm{~m}$ (Fig. 10). The slope 


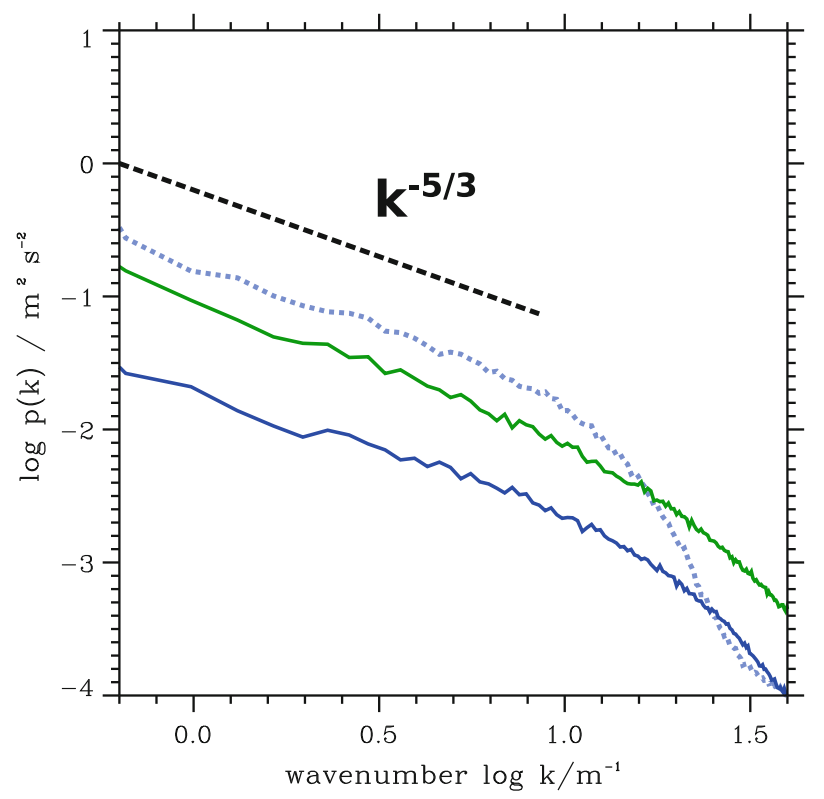

Fig. 10 Power spectra of the resolved TKE at $t=360 \mathrm{~s}$ averaged horizontally at three selected levels located in the trunk space (blue), the crown space (green), and the IBL above the forest canopy (light blue dots)

of the spectrum inside the crown space and trunk space declines slower than at the level inside the IBL above. This spectral behavior at large wave numbers indicates that the wake vortices inject energy into the flow at a scale of the branches of the Pythagoras grove.

\subsection{Diabatic flow phenomena}

A special emphasis of our study was the investigation of the turbulent flow structure of the diabatically heated forest. Diabatic effects in a forest canopy induce buoyancy driven motion and the associated flow can principally be considered as convection in a porous medium [31]. Thereby, coherent structures occur as rising thermals in forest canopies, visible e.g. in Fig. 12 in the LES by Shaw and Schumann [47], and observed in field experiments by [21]. Although numerical modeling of this process is a complex task, resolving the turbulence explicitly helps to understand the flow and its driving forces [45]. Our fractal approach is the prerequisite to investigate the diabatic flow from the plant-scale to the field-scale. Noteworthy, our numerical simulations of the diabatically heated forest generate a mean vertical heat flux profile $\left\langle\theta^{\prime} w^{\prime}\right\rangle$ inside the crown space (Fig. 7) which is in quantitative agreement with a parametric model derived from field observations and applied in previous LESs [3,47].

Basically, and based on the results of our simulations, the forest canopy flow can be divided into three principal layers. We summarize our results in a similar way as done for experimental data by [37]. The first, lowermost layer is called the sub-canopy layer. There, a velocity maximum was simulated (sub-canopy jet) which intensifies for the runs with heated crowns due to the warm rising air that generates a vertical updraft inside the trees resulting in an enhanced wind of the replenishing air near the ground (Fig. 6).

Above the sub-canopy layer, the transition zone inside the crown space constitutes a second principal layer. In this layer, the magnitude of the vertical momentum flux (Fig. 6) and vertical heat flux (Fig. 7) increase until they reach their maxima at the canopy top. In addition, Hovmoeller diagrams of streamwise velocity $u$, sweep and ejection events (Fig. 2) and potential temperature (Fig. 5) reveal the evolution of the turbulent mixing layers for the different stratifications over time.

A third layer evolves above the forest: a shear layer characterized by a gradual increase of IBL height in course of the simulation. This IBL growth is due to turbulent mixing. It was found that the friction velocity $u_{*}$ intensifies due to diabatic heating (Table 2). In field experiments, the IBL is typically observed up to a height of $z<2 h$; c.f. Fig. 2 in [3]. In our simulations, the flow is influenced by the presence of the forest canopy up to a height of $z \approx 4 h$; see e.g. the heat flux profiles in Fig. 7 . 


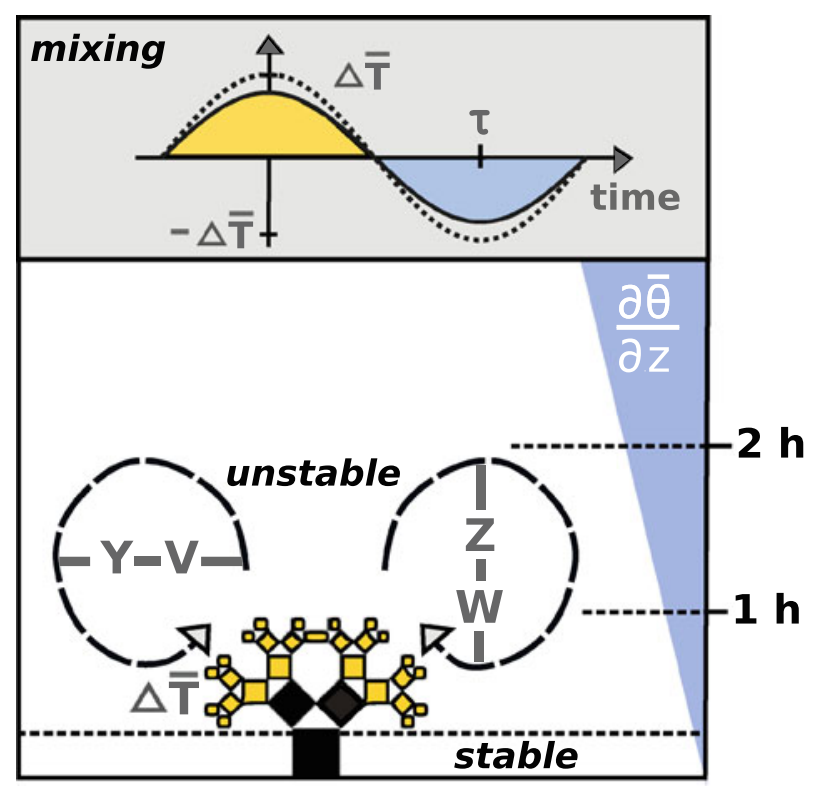

Fig. 11 Sketch of the thermal vortices developing under diabatic heating. Essential parameters determining their strength: background stratification $\bar{N} \sim \partial \bar{\theta} / \partial z$, where $\bar{\theta}$ is time-dependent potential temperature evolving during the simulation, and the temperature deviation $\Delta \bar{T}$ of the heated crowns (yellow shaded). Characteristic parameters of the thermal vortex structures are the vertical extension $Z$, the horizontal extension $Y$, the horizontal velocity $V$, and vertical velocity $W$ for a given eddy turnover time $\tau$. A parcel circulating in the thermal vortex (thick dashed line) experiences the buoyancy force, which varies as ambient air entrains into the air parcel (mixing, top of schematic)

Altogether, the total mixing layer height is determined by two physical processes: first of all, the mechanically driven mixing layer at the canopy top (Kelvin Helmholtz instability) [17] under neutral and stable conditions. Secondly, when the tree crowns are warmer than the ambient air (as in runs 2,3,5), a convectively driven mixing layer (Fig. 6) above the canopy evolves which reminds of a Rayleigh-Taylor instability. This means, the results of our numerical simulations reveal an additional physical process responsible for the growth of the mixing layer above the forest canopy. Eventually, this leads to a modified concept for the mixing layer evolution in contrast to that developed in analogy to a rough wall boundary layer by [37].

In the convective mixing layer coherent circulations arise in the numerical simulations. These thermal vortices even appear in the long-term average $\langle w\rangle_{t}\left(x_{0}, y, z\right)$ as coherent patterns in the $y$-z-plane (Fig. 9b). These circulations are driven by the heterogeneously distributed heated fractal tree crowns. It is worthwhile to sketch a simple schematic of the flow pattern that illustrates the characteristic vertical depth $Z$, the horizontal extension $Y$, the vertical velocity $W$, and the horizontal velocity $V$ of the thermal vortices.

Characteristic parameters determining the circulation are the background stratification $\partial \bar{\theta} / \partial z$ and the temperature difference $\Delta \bar{T}$ of the tree crowns (Fig. 11, bottom). Crucial is the temperature difference of an air parcel located directly above the warm tree crowns $\Delta \bar{T}$. Due to buoyancy such an air parcel is accelerated vertically and a warm updraft forms. The rising air mixes with the environment and the air parcel cools. The temporal evolution of the air parcels temperature difference to the local environment is sketched in the upper panel of Fig. 11. At the uppermost point at $z \approx 2 h$, the unstable stratification cools the air parcel that intrudes into the layer with relatively warmer air and the air parcel sinks. Due to mass conservation and buoyancy force, the relatively cold descending air parcels next to the warm updraft form the closed circulation of the thermal vortex. A quantitative estimation of the characteristic scales if the thermal circulation is complicated due to the presence of dynamic pressure gradients in the flow.

The existence of thermal vortices above heated forests was also suggested by [58]. Furthermore, [61] found that the flow structure in the atmospheric boundary layer is more organized in the late morning hours, when the air is typically unstably stratified. Thereby, [58] found the time scale of $60 \mathrm{~s}$ as a local maximum in the wavelet variance spectrum, where thermal vortices occur most frequently. This time scale corresponds to our estimated eddy turnover time of $\tau=Z / W \approx 30$ s with $Z$ and $W$ as estimated from the $\langle w\rangle_{t}\left(x_{0}, y, z\right)$-values in the updrafts of our numerical simulation. It is important to note, that our eddy turnover time $\tau$ is the time, an air parcel needs to travel around half of the vortex. 
Future studies in our LES will focus on flow phenomena at the plant-scale, include moist processes, and a higher spatial resolution. Furthermore, the impact of a larger heterogeneous forest on the stratified flow will be investigated.

Acknowledgments The computing time at the high performance computing platform of the DKRZ was provided through the project "Multiskalen-Simulationen mit EULAG". We thank Björn Brötz, Christian Kühnlein, Takashi Misaka, and Dieter Heimann for comments on an early version of the paper. The work formed part of a master thesis at the Ludwig-Maximilians-Universität (LMU) Munich in collaboration with the German Aerospace Center (DLR). It was supported by the Oskar Karl Forster scholarship and a travel grant by DAAD (German Academic Exchange Service). Especially, we thank Piotr K. Smolarkiewicz for many helpful discussions and for hosting JS during his three month visit at NCAR. We appreciate the implementation of the online statistics by Christian Kühnlein.

\section{Appendix A: Topological measures for forest canopies}

The leaf area density $\operatorname{LAD}(x, y, z)$ of a forest canopy is a measure for the local density of vegetation inside the forest canopy at position $\mathbf{x}=(x, y, z)$. Therefore, the relative volume occupied with e.g. trunks, branches, or foliage is determined. The three-dimensional LAD was investigated in field campaigns as in [42]. They generated a 3D, so called voxel space, where the relative amount of vegetation was represented as $\operatorname{LAD}(x, y, z)=$ vegetation area / surface area from vegetation laser scans.

In our study, the Pythagoras grove (Fig. 1) is the discretely resolved geometrical representation of the wooden trunks, branches, or the foliage. In the computational domain, we set $\operatorname{LAD}(x, y, z)>0$, if a solid obstacle occupies a grid box.

To compare the LAD of different forest canopies with a single number, the leaf area index (LAI) can be calculated. Thereby, the LAD is a dimensionless scalar field and the heaviside step function $H$ indicates the presence of a solid obstacle:

$$
\mathrm{LAI}=\int_{0}^{L_{x}} \int_{0}^{L_{y}} \int_{0}^{L_{z}} H[\operatorname{LAD}(x, y, z)] \mathrm{d} \tilde{x} \mathrm{~d} \tilde{y} \mathrm{~d} \tilde{z}=\frac{1}{n} \frac{1}{m} \frac{1}{l} \sum_{i, j, k} H[\operatorname{LAD}(i, j, k)]
$$

In addition $\tilde{x}=x / L_{x}, \tilde{y}=y / L_{y}$, and $\tilde{z}=z / L_{z}$ are the normalized dimensionless length scales. In the numerical domain, the LAI is calculated as a sum over the grid increments $(i, j, k) \in[0, n] \times[0, m] \times[0, l]$ and normalized by their number. The LAI is e.g. lower during partial leaf fall and has typical values of 1.6 [46], 2 and $5[12,47]$.

\section{Appendix B: Numerical construction of a Pythagoras tree}

The history of the fractal Pythagoras tree dates back to 1952, when the German mathematician A. E. Bosman (1891-1961) constructed it [14]. The Pythagoras tree can mimic a wide range of real world trees of different complexity ${ }^{7}$-as its shape only depends on the types of right triangles that are used for its construction. We use a very simple fractal Pythagoras tree with only isosceles right triangles, which fully suits our purpose as a basis for the numerical forest model (Fig. 1). The goal is to resolve the trees at the scale of individual plant feature, the so called plant-scale.

To construct the trees in two dimensions, the simple recursive IDL algorithm ${ }^{8}$ proceeds in the following way: an isosceles right triangle with the baseline length $l_{0}=\overline{A B}$ is placed on a rectangle representing the trunk (Fig. 12), which is the initiator for the algorithm. The two other sides of the triangle are equal in length and amount to $l_{0} / \sqrt{2}$. They constitute the baselines for two new squares placed on their legs $[D, T]$ and

\footnotetext{
7 E.g. see pp. 127-128, Fig. 2.65, 2.66 and 2.67 in 'Chaos and Fractals' by Peitgen et al. Springer (2004) [34].

${ }^{8}$ Our IDL code is available in the online publication at http://www.springerlink.com/content/0935-4964.
} 


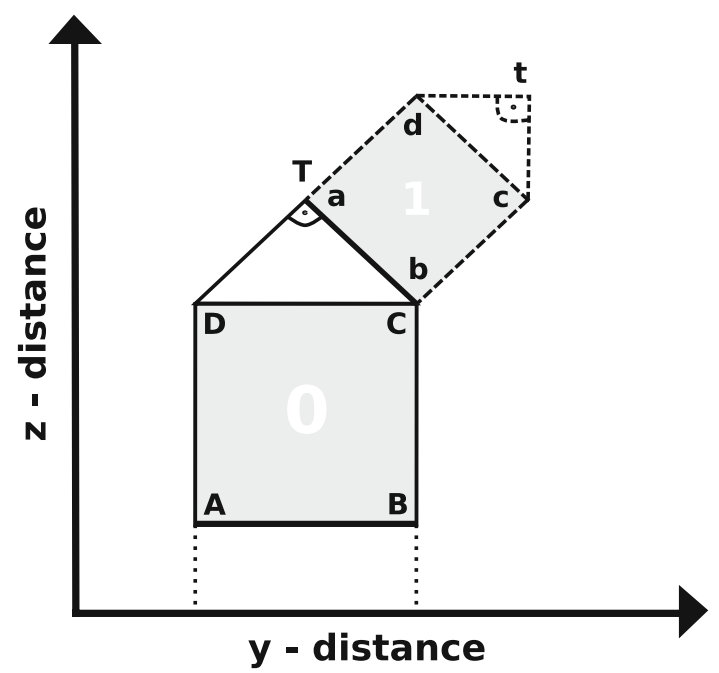

Fig. 12 Construction of a Pythagoras tree: the initiator, a square with points $A, B, C$ and $D$ is set on top of a rectangle (dotted line), where $[A, B]$ forms the baseline (heavy solid line) of the initiator. Then, a new square with points $a, b, c$ and $d$ (dashed line) is initiated on the right leg $[T, C]$ of an isosceles right triangle with points $A, C, T$. The baseline $[a, b]$ of the new square or branch is $[T, C]$. For each new generation $f$ (white number), $2^{f}$ branches are initiated recursively and a self-similar, fractal structure evolves (Fig. 1)

$[T, C]$. The new coordinates e.g. for the right branch: $a, b, c$ and $d$, are computed from the baseline $[T, C]$ with $a=T, b=C$ as follows: ${ }^{9}$

$$
\begin{array}{r}
c=\left(\begin{array}{l}
b_{y}+a_{z}-b_{z} \\
b_{z}+b_{y}-a_{y}
\end{array}\right) \\
d=\left(\begin{array}{l}
a_{y}+a_{z}-b_{z} \\
a_{z}+b_{y}-a_{y}
\end{array}\right)
\end{array}
$$

The algorithm continues by placing again an isosceles triangle; where $r_{1}=r_{2}=0.5$, on top of each square e.g. for the right branch with coordinates $d, t$ and $c$ :

$$
t=\left(\begin{array}{c}
d_{y}+r_{1}\left(c_{y}-d_{y}\right)+r_{2}\left(a_{z}-b_{z}\right) \\
d_{z}+r_{1}\left(c_{z}-d_{z}\right)+r_{2}\left(b_{y}-a_{y}\right)
\end{array}\right)
$$

This can be repeated down to an arbitrary branch generation $\mathrm{n}$. Each tree constructed in this way has fractal dimension [28] of $D=\log (N(l)) / \log (1 / r(l))=\log (2) / \log (\sqrt{2})=2$, where $N(l)$ is the number of branches spreading from the new baseline with a ratio of $r(l)$ smaller than the last generation. Thus, generation $\mathrm{f}$ has a baseline of length $l_{f}=l_{f-1} / \sqrt{2}$ and a number of $2^{f}$ branches. In our numerical algorithm all squares are filled and triangles are spared. Expanding the structure in 3D, with a certain depth in $x$-direction (Fig. 1), provides the basis for a fractal Pythagoras tree, as it is used in our numerical forest.

\section{Appendix C: Online statistics}

Temporal averages \langle\rangle$_{t}$ over 10 and $20 \mathrm{~s}$ were computed at every grid point for each new time $t^{n+1}$ according to

$$
\langle\psi\rangle_{t}^{n+1}=\epsilon \psi^{n+1}+(1-\epsilon)\langle\psi\rangle_{t}^{n}
$$

and

$$
\left\langle\psi^{\prime} \phi^{\prime}\right\rangle_{t}^{n+1}=\epsilon\left(\psi^{n+1}-\langle\psi\rangle_{t}^{n+1}\right)\left(\phi^{n+1}-\langle\phi\rangle_{t}^{n+1}\right)+(1-\epsilon)\left\langle\psi^{\prime} \phi^{\prime}\right\rangle_{t}^{n}
$$

\footnotetext{
9 Adopted from a java applet: http://www.jjam.de/Java/Applets/Fraktale/Pythagoras_Baum.html.
} 
where $\psi, \phi=u, v, w, \theta$, and $\epsilon=\Delta t /\left(t^{n}-t^{\text {start }}\right)$ with $t^{n}$ the time of the $n$th time step and $t^{\text {start }}=$ $60 \mathrm{~s}, 80 \mathrm{~s}, \ldots, 340 \mathrm{~s}$. This online statistical evaluation follows [20], p. 273. It can be used in numerical simulations with a time step that varies throughout the simulation, e.g. in combination with adaptive moving meshes [27].

\section{References}

1. Baldocchi, D.D. et al.: Predicting the onset of net carbon uptake by deciduous forests with soil temperature and climate data: a synthesis of FLUXNET data. Int. J. Biometeorol. 6, 377-387 (2005)

2. Belcher, S.E., Harman, I.N., Finnigan, J.J.: The wind in the willows: flows in forest canopies in complex terrain. Ann. Rev. Fluid Mech. 44, 479-504 (2012)

3. Bohrer, G., Katul, G.G., Walko, R.L., Avissar, R.: Exploring the effects of microscale structural heterogeneity of forest canopies using large-eddy simulations. Bound. Layer Meteorol. 132, 351-382 (2009)

4. Bou-Zeid, E., Meneveau, C., Parlange, M.B.: Large-eddy simulation of neutral atmospheric boundary layer flow over heterogeneous surfaces: blending height and effective surface roughness. Water Resour. Res. 40, 2 (2004)

5. Brown, K.W., Covey, W.: The energy-budget evaluation of the micro-meteorological transfer process within a cornfield. J.Agric. Meteorol. 3, 13-96 (1966)

6. Brunet, Y., Finnigan, J.J., Raupach, M.R.: A wind tunnel study of air flow in waving wheat: single-point velocity statistics. Bound. Layer Meteorol. 70, 95-132 (1994)

7. Campos, J.G., Acevedo, O.C., Tota, J., Manzi, A.O.: On the temporal scale of the turbulent exchange of carbon dioxide and energy above a tropical rain forest in Amazonia. J. Geophys. Res. 114, D08124 (2009)

8. Cava, D., Katul, G.G.: Spectral short-circuiting and wake production within the canopy trunk space of an alpine hardwood forest. Bound. Layer Meteorol. 126, 415-431 (2008)

9. Chester, S., Meneveau, C., Parlange, M.: Modeling turbulent flow over fractal trees with renormalized numerical simulation. J.Comput. Phys. 225, 427-448 (2007)

10. de Langre, E.: Effects of wind on plants. Annu. Rev. Fluid Mech. 40, 141-168 (2008)

11. Doyle, J.D. et al.: An intercomparison of T-REX mountain wave simulations and implications for mesoscale predictability. Mon. Weather Rev. 139, 2811-2831 (2011)

12. Dupont, S., Brunet, Y.: Coherent structures in canopy edge flow: a large-eddy simulation study. J. Fluid Mech. 630, 93-127 (2009)

13. Dupont, S., Irvine, M.R., Bonnefond, J.M., Lamaud, E., Brunet, Y.: Turbulent Structures in a Pine Forest with a Deep and Sparse Trunk Space: Stand and Edge Regions. Bound. Layer Meteorol. 143, 309-336 (2012)

14. Elaydi, S.N.: Discrete Chaos: With Applications in Science and Engineering. Chapman \& Hall, New York (2008)

15. Finnigan, J.J.: Turbulence in plant canopies. Annu. Rev. Fluid Mech. 32, 519-571 (2000)

16. Finnigan, J.J., Shaw, R.: A wind-tunnel study of airflow in waving wheat: an EOF analysis of the structure of the large-eddy motion. Bound. Layer Meteorol. 96, 211-255 (2000)

17. Finnigan, J.J., Shaw, R.H., Patton, E.G.: Turbulence structure above a vegetation canopy. J. Fluid Mech. 637, 387-424 (2009)

18. Foken, T., Wimmer, F., Mauder, M., Thomas, C., Liebethal, C.: Some aspects of the energy balance closure problem. Atmos. Chem. Phys. 6, 4395-4402 (2006)

19. Frisch, U.: Turbulence: The Legacy of A. N. Kolmogorov. Cambridge University Press, Cambridge (2009)

20. Fröhlich, J.: Large Eddy Simulation Turbulenter Strömungen. Vieweg \& Teubner Verlag, Wiesbaden (2006)

21. Gao, W., Shaw, R.H., Paw, K.T.: Observation of organized structure in turbulent flow within and above a forest canopy. Bound. Layer Meteorol. 47, 349-377 (1989)

22. Goldstein, D., Handler, R., Sirovich, L.: Modeling a no-slip flow boundary with an external force field. J. Comput. Phys. 105, 354-366 (1993)

23. Gross, G.: A numerical study of the air flow within and around a single tree. Bound. Layer Meteorol. 40, 311-327 (1987)

24. Jeong, J., Hussain, F.: On the identification of a vortex. J. Fluid Mech. 285, 69-94 (1995)

25. Katul, G., Kuhn, G., Schieldge, J., Hsieh, C.I.: The ejection-sweep character of scalar fluxes in the unstable surface layer. Bound. Layer Meteorol. 83, 1-26 (1997)

26. Kundu, P.K., Cohen, I.M.: Fluid Mechanics. Academic Press, Elsevier (2008)

27. Kühnlein, C., Smolarkiewicz, P.K., Dörnbrack, A.: Modelling atmospheric flows with adaptive moving meshes. J. Comput. Phys. 231, 2741-2763 (2012)

28. Mandelbrot, B.: The Fractal Geometry of Nature. W. H. Freeman, New York (1983)

29. Margolin, L.G., Smolarkiewicz, P.K., Sorbjan, Z.: Large-eddy simulations of convective boundary layers using nonoscillatory differencing. Phys. D. 133, 390-397 (1999)

30. Mittal, R., Iaccarino, G.: Immersed boundary methods. Ann. Rev. Fluid Mech. 37, 239-261 (2005)

31. Nield, D.A., Bejan, A.: Convection in Porous Media. Springer, Berlin (2006)

32. Niinemets, Ü., Seufert, G., Steinbrecher, R., Tenhunen, J.D.: A model coupling foliar monoterpene emissions to leaf photosynthetic. New Phytol. 153, 257-275 (2002)

33. Patton, E.G. et al.: The canopy horizontal array turbulence study. Bull. Am. Meteorol. Soc. 92, 593-611 (2011)

34. Peitgen, H.O., Jürgens, H., Saupe, D.: Chaos and Fractals, New Frontiers of Science. Springer, New York (2004)

35. Piotrowski, Z.P., Smolarkiewicz, P.K., Malinowski, S.P., Wyszogrodzki, A.A.: On numerical realizability of thermal convection. J. Comput. Phys. 228, 6268-6290 (2009)

36. Piotrowski, Z.P., Wyszogrodzki, A.A., Smolarkiewicz, P.K.: Towards petascale simulation of atmospheric circulations with soundproof equations. Acta Geophys. 59, 1294-1311 (2011)

37. Poggi, D., Porporato, A., Ridolfi, L., Albertson, J.D., Katul, G.G.: The effect of vegetiation density on canopy sub-layer turbulence. Bound. Layer Meteorol. 111, 565-587 (2004) 
38. Prusa, J.M., Smolarkiewicz, P.K.: An all-scale anelastic model for geophysical flows: dynamic grid deformation. J. Comput. Phys. 190, 601-622 (2003)

39. Prusa, J.M., Smolarkiewicz, P.K., Wyszogrodzki, A.A.: EULAG, a computational model for multiscale flows. Comput. Fluids 37, 1193-1207 (2008)

40. Poveda-Jaramillo, G., Puente, C.E.: Strange attractors in atmospheric boundary-layer turbulence. Bound. Layer Meteorol. 64, 175-197 (1993)

41. Raupach, M.R., Coppin, P.A., Legg, B.J.: Experiments on scalar dispersion within a model plant canopy part I: the turbulence structure. Bound. Layer Meteorol. 35, 21-52 (1986)

42. Schlegel, F., Stiller, J., Bienert, A., Maas, H.G., Queck, R., Bernhofer, C.: Large-Eddy Simulation of Inhomogeneous Canopy Flows Using High Resolution Terrestrial Laser Scanning Data. Bound. Layer Meteorol. 142, 1-21 (2012)

43. Schmitt, R.W.: The salt finger experiments of Jevons (1857) and Rayleigh (1880). J. Phys. Oceanogr. 25, 8-17 (1995)

44. Schmitt, R.W., Ledwell, J.R., Montgomery, E.T., Polzin, K.L., Toole, J.M.: Enhanced diapycnal mixing by salt fingers in the thermocline of the tropical Atlantic. Science 308, 685 (2005)

45. Schulze, T.P., Worster, M.G.: Weak convection, liquid inclusions and the formation of chimneys in mushy layers. J. Fluid Mech. 388, 197-215 (1999)

46. Shaw, R.H., Hartog, G., Neumann, H.H.: Influence of foliar density and thermal stability on profiles of Reynolds stress and turbulence intensity in a deciduous forest. Bound. Layer Meteorol. 45, 391-409 (1988)

47. Shaw, R., Schumann, U.: Large-eddy simulation of turbulent-flow above and within a forest. Bound. Layer Meteorol. 61, 47-64 (1992)

48. Smolarkiewicz, P.K., Margolin, L.G.: On forward-in-time differencing for fluids: extension to a curvilinear framework. Mon. Weather Rev. 121, 1847-1859 (1993)

49. Smolarkiewicz, P.K., Margolin, L.G.: MPDATA: a finite-difference solver for geophysical flows. J. Comput. Phys. 140, 459-480 (1998)

50. Smolarkiewicz, P.K., Prusa, J.M.: VLES modelling of geophysical fluids with nonoscillatory forward-in-time schemes. Int. J. Numer. Methods Fluids. 39, 799-819 (2002)

51. Smolarkiewicz, P.K.: Multidimensional positive definite advection transport algorithm: an overview. Int. J. Numer. Methods Fluids. 50, 1123-1144 (2006)

52. Smolarkiewicz, P.K., Margolin, L.G. : Studies in geophysics. In: Grinstein, F.F., Margolin, L.G., Rider, W. (eds.) Implicit Large Eddy Simulation: Computing Turbulent Fluid Dynamics, pp. 413-438. Cambridge University Press, Cambridge (2007)

53. Smolarkiewicz, P.K., Sharman, R., Weil, J., Perry, S., Heist, D., Bowker, G.: Building resolving large-eddy simulations and comparison with wind tunnel experiments. J. Comput. Phys. 227, 633-653 (2007)

54. Smolarkiewicz, P.K., Winter, C.: Pores resolving simulation of Darcy flows. J. Comput. Phys. 229, 3121-3133 (2010)

55. Staebler, R.M., Fitzjarrald, D.R.: Measuring canopy structure and the kinematics of subcanopy flows in two forests. J. Appl. Meteorol. Climatol. 44, 1161-1179 (2005)

56. Stull, R.B.: An Introduction to Boundary Layer Meteorology. Kluwer Academic Publishers, Netherlands (1988)

57. Su, Z. et al.: EAGLE 2006-multi-purpose, multi-angle and multi-sensor in-situ and airborne campaigns over grassland and forest. Hydrol. Earth Syst. Sci. 13, 833-845 (2003)

58. Thomas, C., Mayer, J.C., Meixner, F.X., Foken, T.: Analysis of low-frequency turbulence above tall vegetation using a Doppler sodar. Bound. Layer Meteorol. 119, 563-587 (2006)

59. Yue, W., Parlange, M.B., Meneveau, C., Zhu, W., Van Hout, R., Katz, J.: Large-eddy simulation of plant canopy flows using plant-scale representation. Bound. Layer Meteorol. 124, 183-203 (2007)

60. Wedi, N.P., Smolarkiewicz, P.K.: Extending Gal-Chen and Somerville terrain-following coordinate transformation on time dependent curvilinear boundaries. J. Comput. Phys. 193, 1-20 (2004)

61. Wesson, K.H., Katul, G.G., Siqueira, M.: Quantifying organization of atmospheric turbulent eddy motion using nonlinear time series analysis. Bound. Layer Meteorol. 106, 507-525 (2003) 